\title{
Dynamics of a degenerate Cs-Yb mixture with attractive interspecies interactions
}

\author{
Kali E. Wilson $\odot,{ }^{1, *}, \dagger$ Alexander Guttridge $\odot,{ }^{1,}{ }^{*}$ I-Kang Liu $\odot,{ }^{2}$ Jack Segal $\odot,{ }^{1}$ Thomas P. Billam $\odot,{ }^{2}$ Nick G. Parker $\odot,{ }^{2}$ \\ N. P. Proukakis $\odot, 2$ and Simon L. Cornish $\oplus^{1, 末}$ \\ ${ }^{1}$ Joint Quantum Centre Durham-Newcastle, Department of Physics, Durham University, South Road, Durham DH1 3LE, United Kingdom \\ ${ }^{2}$ Joint Quantum Centre Durham-Newcastle, School of Mathematics, Statistics and Physics, Newcastle University, \\ Newcastle upon Tyne NE1 7RU, United Kingdom
}

(Received 18 December 2020; accepted 9 June 2021; published 27 July 2021)

\begin{abstract}
We probe the collective dynamics of a quantum degenerate Bose-Bose mixture of ${ }^{133} \mathrm{Cs}$ and ${ }^{174} \mathrm{Yb}$ with attractive interspecies interactions. Specifically, we excite vertical center-of-mass oscillations of the Cs condensate. We observe significant damping for the Cs dipole mode, due to the rapid transfer of energy to the larger $\mathrm{Yb}$ component and the ensuing acoustic dissipation. Numerical simulations based on coupled Gross-Pitaevskii equations provide excellent agreement with experiment and additionally reveal the possibility of late-time revivals (beating), which are found to be highly sensitive to the $\mathrm{Cs}$ and $\mathrm{Yb}$ atom number combinations. By further tuning the interaction strength of $\mathrm{Cs}$ using a broad Feshbach resonance, we explore the stability of the degenerate mixture. We observe collapse of the $\mathrm{Cs}$ condensate mediated by the attractive $\mathrm{Cs}$ - $\mathrm{Yb}$ interaction when $a_{\mathrm{Cs}}<50 a_{0}$, well above the single-species collapse threshold, in good agreement with simulations.
\end{abstract}

DOI: 10.1103/PhysRevResearch.3.033096

\section{INTRODUCTION}

Superfluid mixtures formed of ultracold atoms offer a versatile playground for studies of binary fluid dynamics in both Bose-Bose and Bose-Fermi mixtures. Interplay between superfluids can lead to counterflow instabilities [1-4] and quantum turbulence $[1,5]$. Previous experimental work has explored collective excitations in the form of scissors mode oscillations [6] in repulsive Bose-Bose mixtures; spin dynamics in spinor Bose-Einstein condensates (BECs) with repulsive interactions [7]; and dipolar oscillations [8], breathing dynamics [9], and the exchange of angular momentum [10] in Bose-Fermi mixtures. The role of immiscibility and temperature in the expansion dynamics of repulsive Bose-Bose mixtures has also been investigated [11]. Experimental control in ultracold atomic mixtures allows the balance of the interand intraspecies interactions to be tuned, enabling further manipulation of the coupled dynamics and stability of the system. This can result in novel bound states such as bright-dark solitons [12] and exotic vortex lattices $[13,14]$ and enable miscibility studies [15]. Prior research on dynamical instabilities associated with attractive interspecies interactions has focused

\footnotetext{
*These authors contributed equally to this work.

${ }^{\dagger}$ Present address: Department of Physics, SUPA, University of Strathclyde, Glasgow G4 0NG, United Kingdom.

‡s.1.cornish@durham.ac.uk

Published by the American Physical Society under the terms of the Creative Commons Attribution 4.0 International license. Further distribution of this work must maintain attribution to the author(s) and the published article's title, journal citation, and DOI.
}

on Bose-Fermi mixtures, where the bosonic species mediates collapse and subsequent density-dependent atom loss in the fermionic species [16-18]. However, recent studies have used Bose-Bose mixtures to probe the region where the net meanfield interaction is close to zero, resulting in the observation of quantum droplets where quantum fluctuations provide a repulsive force that stabilizes against collapse [19-21].

In this paper, we probe the collective dynamics and stability of a quantum degenerate Bose-Bose mixture of ${ }^{133} \mathrm{Cs}$ and ${ }^{174} \mathrm{Yb}$ with an attractive interspecies interaction characterized by the scattering length $a_{\mathrm{CsYb}}=-75 a_{0}$ [22]. The Cs intraspecies scattering length $a_{\mathrm{Cs}}$ is a tunable experimental parameter via a broad, low-field magnetic Feshbach resonance [23,24], while the ${ }^{174} \mathrm{Yb}$ intraspecies scattering length is $a_{\mathrm{Yb}}=105 a_{0}$. We use vertical Cs center-of-mass (CoM) oscillations to probe the collective dynamics of the system, observing an upwards mean-field shift of the CoM dipole mode frequency. We also find that the attractive interspecies interaction results in complicated coupled dynamics, as $\mathrm{Cs}$ pulls along $\mathrm{Yb}$ in the region of $\mathrm{Cs}-\mathrm{Yb}$ overlap. We probe the stability of the mixture by tuning the Cs interaction strength across the collapse threshold, demonstrating Yb-mediated collapse of the Cs BEC, well above the single-species collapse threshold.

\section{EXPERIMENTAL OVERVIEW}

We prepare dual-degenerate mixtures of ${ }^{133} \mathrm{Cs}$ in the $\left|F=3, m_{F}=+3\right\rangle$ hyperfine state and ${ }^{174} \mathrm{Yb}$ using the apparatus and procedures described in Refs. [25-29]. Our dual-species evaporation relies on sympathetic cooling of $\mathrm{Cs}$ with $\mathrm{Yb}$, resulting in an imbalanced mixture with typical condensate atom numbers of $\left(N_{\mathrm{Yb}}, N_{\mathrm{Cs}}\right)=(50,5) \times 10^{3}$ with 
(a)

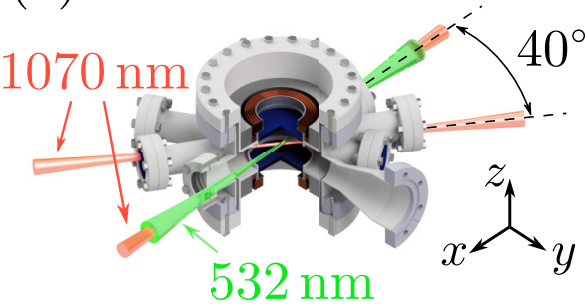

(b)

(c)

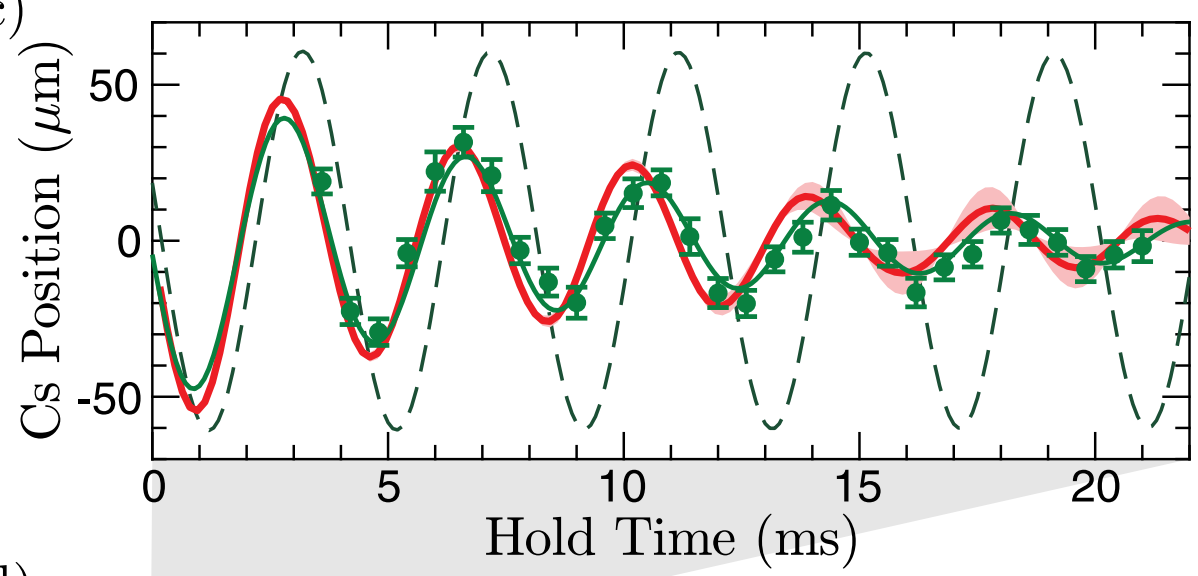

(d)

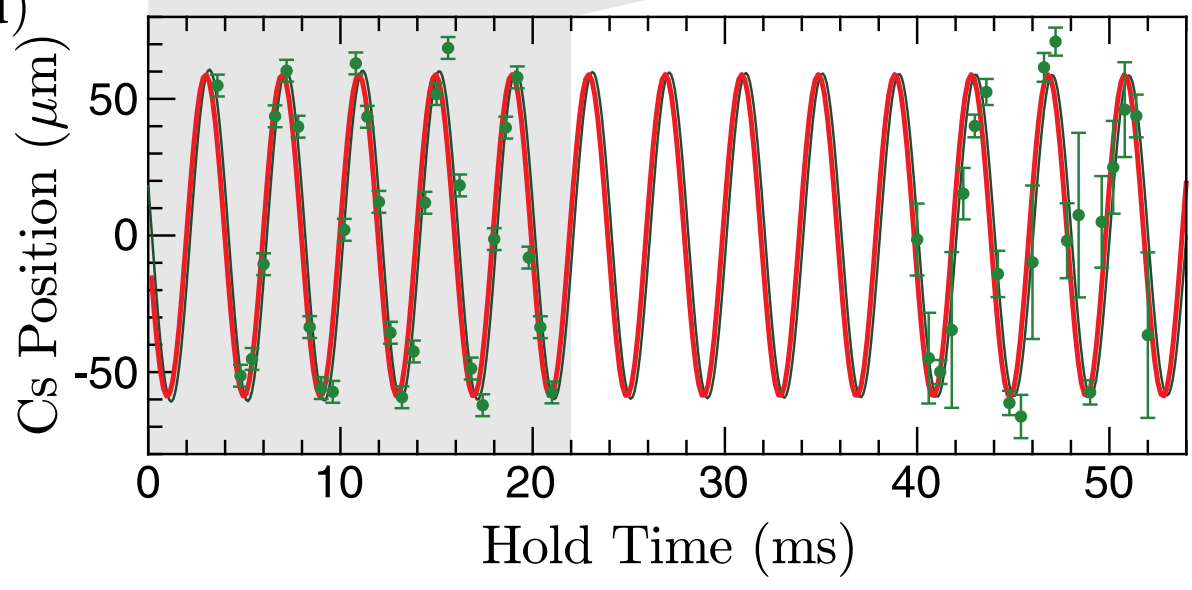

FIG. 1. Cs and Yb coupled dynamics. (a) Layout of the bichromatic optical dipole trap. (b) Numerically generated coupled displaced $3 \mathrm{D}$ density profiles for $\mathrm{Cs}$ (red) and $\mathrm{Yb}$ (blue) upon introducing a $+0.95-\mu \mathrm{m}$ vertical shift of the Cs trap minimum (at time $t=0$ ) with $\left(N_{\mathrm{Yb}}, N_{\mathrm{Cs}}\right)=(40,6.5) \times 10^{3}$. Solid lines are sliced density profiles along the $x, y$, and $z$ axes. (c) Vertical CoM position of the Cs BEC after $40 \mathrm{~ms}$ ToF vs hold time in the trap measured experimentally (green circles) and simulated numerically (red line). The solid green line is a fit to the experiment: $A e^{-\beta t} \sin \left(\omega t+\phi_{0}\right)$ with $A=52(8) \mu \mathrm{m}, \omega=2 \pi \times 260(2) \mathrm{Hz}$, and $\beta=0.10(2) \mathrm{ms}^{-1}$. The numerical plot is based on an average over 204 particle number sets, with the red band indicating the standard deviation. Atom numbers are randomly selected such that their mean is $\left(N_{\mathrm{Yb}}, N_{\mathrm{Cs}}\right)=(40,6.5) \times 10^{3}$, with a $20 \%$ standard deviation. (d) Corresponding CoM position of the Cs BEC performed in the absence of $\mathrm{Yb}$, yielding $\omega=2 \pi \times 251$ (1) Hz. The dashed green line in (c) reproduces the fit to the measurement shown in (d).

up to $20 \%$ shot-to-shot variation [29]. The condensates are confined in a bichromatic optical dipole trap (BODT) [30], using laser beams with wavelengths of 532 and $1070 \mathrm{~nm}$ aligned as shown in Fig. 1(a). We define a coordinate system $(x, y, z)$ with $x$ along the copropagating BODT beams and $z$ being the vertical direction. The axes $\tilde{x}$ and $\tilde{y}$ for the Cs trap are rotated $20^{\circ}$ from the $\mathrm{Yb}$ trap axes; the rotation of the $\tilde{x}$ (weak) and $\tilde{y}$ trap axes results from the differences in Cs and $\mathrm{Yb}$ polarizabilities at the BODT wavelengths. The final trap frequencies are $\left(\omega_{\mathrm{x}}, \omega_{\mathrm{y}}, \omega_{\mathrm{z}}\right)_{\mathrm{Yb}}=2 \pi \times(10,120,80) \mathrm{Hz}$ for
Yb and $\left(\omega_{\tilde{x}}, \omega_{\tilde{y}}, \omega_{\mathrm{z}}\right)_{\mathrm{Cs}}=2 \pi \times(40,70,260) \mathrm{Hz}$ for Cs. The vertical trap frequencies are very sensitive to the overlap of the BODT beams and can vary by up to $10 \%$ with day-to-day realignment of the trap [29].

\section{COUPLED DYNAMICS}

\section{A. Center-of-mass oscillations}

We explore the coupled dynamics of the mixture by exciting Cs center-of-mass (CoM) oscillations in the vertical 
direction. At the end of the dual-species evaporation sequence we adiabatically ramp on a magnetic field gradient of $9.0 \mathrm{G} / \mathrm{cm}$ in the $z$ direction. This additional magnetic potential causes a $0.96(7)-\mu \mathrm{m}$ vertical shift of the Cs trap minimum, without directly acting on $\mathrm{Yb}$, which has no magnetic moment. The gradient is rapidly switched off, exciting the vertical CoM dipole mode. We wait a variable hold time before turning off the trapping potential and then perform absorption imaging of the Cs condensate after $40 \mathrm{~ms}$ of levitated time of flight (ToF). We hold $a_{\mathrm{Cs}}$ constant at $275 a_{0}$ for both in-trap oscillations and the subsequent ToF. To account for the high sensitivity of the vertical trap frequencies to alignment, we reference the coupled $\mathrm{Cs}-\mathrm{Yb}$ dynamics to the Cs CoM oscillations in the absence of $\mathrm{Yb}$ by taking both measurements in quick succession. We obtain the Cs-only reference by selectively removing $\mathrm{Yb}$ with a 5-ms pulse of light resonant with the ${ }^{1} S_{0} \rightarrow{ }^{1} P_{1}$ transition at $399 \mathrm{~nm}$ prior to exciting the Cs CoM oscillations. To gain further insight into the rich oscillatory dynamics, our experimental measurements are supplemented by numerical simulations based on coupled three-dimensional (3D) Gross-Pitaevskii equations (GPEs) (see Appendixes A and B). A numerical visualization of the initial geometry with $\left(N_{\mathrm{Yb}}, N_{\mathrm{Cs}}\right)=(40,6.5) \times 10^{3}$ atoms, $\omega_{\mathrm{Cs}, z}=2 \pi \times 250 \mathrm{~Hz}$, and an imposed $+0.95-\mu \mathrm{m}$ vertical shift of the Cs trap position is shown in Fig. 1(b).

The measured vertical CoM oscillations of the Cs BEC in the presence of the $\mathrm{Yb}$ BEC are shown by the green circles in Fig. 1(c). These oscillations are strikingly different from the corresponding Cs-only oscillations shown in Fig. 1(d) [also dashed green line in Fig. 1(c)], which occur at the Cs natural trap frequency $\omega_{\mathrm{Cs}, z}=2 \pi \times 251(1) \mathrm{Hz}$ with minimal damping. In the presence of $\mathrm{Yb}$, the $\mathrm{Cs} \mathrm{CoM}$ oscillations experience a frequency shift upwards of 4(1)\%, with significant damping. This shift is in good agreement with a first-order mean-field prediction that assumes a Thomas-Fermi profile for the $\mathrm{Yb}$ $\mathrm{BEC}$ and neglects the back action of the $\mathrm{Cs} \mathrm{BEC}$ on the $\mathrm{Yb}$ atoms due to the large number imbalance (see Appendix B 1). Under such assumptions, the interspecies interaction results in an additional attractive quadratic potential for Cs that leads to a $5 \%$ upwards frequency shift.

The numerical simulations reveal that both components start the coupled dynamics from nonequilibrium positions with respect to their trap centers. The attractive $\mathrm{Cs}-\mathrm{Yb}$ interaction induces a density "bulge" in the $\mathrm{Yb} \mathrm{BEC}$ in the region of Cs- $\mathrm{Yb}$ overlap, visible in the $z$-axis density profile shown in Fig. 1(b). The off-center bulge leads to a vertical $\mathrm{Yb} \mathrm{CoM}$ shift upwards of $\Delta z_{\mathrm{Yb}}^{\mathrm{CoM}}=0.1 \mu \mathrm{m}$. Similarly, the Cs CoM shift reduces to $\Delta z_{\mathrm{Cs}}^{\mathrm{CoM}}=0.88 \mu \mathrm{m}$.

The simulated vertical CoM oscillations of the Cs BEC are shown by the red line in Figs. 1(c) and 1(d) for $\mathrm{Cs}+\mathrm{Yb}$ and $\mathrm{Cs}$ only, respectively. The $\mathrm{Cs}+\mathrm{Yb}$ simulations indicate that the CoM shifts and ensuing coupled dynamics are highly sensitive to the relative $\mathrm{Cs}$ and $\mathrm{Yb}$ atom numbers, with representative plots over a range of atom numbers shown in Appendix B 2. Therefore, to account for shot-to-shot variations in both $\mathrm{Cs}$ and $\mathrm{Yb}$ atom numbers, the simulation result shown in Fig. 1(c) is based on the average of 204 atom number combinations, randomly selected such that their mean and standard deviations are $\left(N_{\mathrm{Yb}}, N_{\mathrm{Cs}}\right)=(40,6.5) \times$ $10^{3}$ and $20 \%$, respectively, in broad agreement with the values measured experimentally. As shown in Fig. 1(c), this leads to excellent agreement with the experimental observations. Representative density snapshots are provided in Appendix B 3, and a video of the coupled dynamics is available in the Supplemental Material [31]. The results from the Cs-only simulations shown in Fig. 1(d) provide excellent agreement with the experiment independent of $\mathrm{Cs}$ atom number. Closer analysis of the simulations reveals that the $\mathrm{Cs}+\mathrm{Yb}$ dynamics are complicated by the different vertical trap frequencies experienced by the two components $\left(\omega_{\mathrm{Cs}, z} \approx 3 \omega_{\mathrm{Yb}, z}\right)$, the large atom number imbalance $\left(N_{\mathrm{Yb}} / N_{\mathrm{Cs}} \approx 6\right)$, and the broader spatial extent of the $\mathrm{Yb}$ BEC in the weakly confined direction. The resulting evolution is thus dominated by the dynamics of the central bulge in the $\mathrm{Yb}$ BEC that directly interacts with the Cs BEC.

\section{B. Damping and excitations}

A striking feature of Fig. 1(c) is the fast damping of the Cs CoM oscillations in the presence of the $\mathrm{Yb}$ BEC, with the amplitude falling to $1 / e$ its initial value in $10(2) \mathrm{ms}$. We explore the origin of this damping in Fig. 2. Figure 2(a) shows the numerical CoM motions out to $180 \mathrm{~ms}$, while Fig. 2(b) shows the evolution of the relevant energies over the same timescale. The gray region shown in both plots indicates the first $22 \mathrm{~ms}$ of evolution, where the primary damping and subsequent energy transfer occur. Looking first at the changes in the $\mathrm{Cs}$ and $\mathrm{Yb}$ energies shown in Fig. 2(b), we see that there is a net loss of $\sim 85 \%$ of the initial excess Cs potential energy over the first $\approx 22 \mathrm{~ms}$. This energy is transferred to the $\mathrm{Yb}$ component through a coupling of the CoM motions to low-lying excitations. Although the initial excitations are produced along the $z$ axis of relative motion, an additional secondary mode coupling between the $z$ and $y$ directions gradually develops owing to the comparable $\mathrm{Yb}$ trap frequencies in these directions. This is evident in Fig. 2(b) as an out-ofphase oscillation in $\Delta E_{\mathrm{Yb}, y}^{0}$ and $\Delta E_{\mathrm{Yb}, z}^{0}$, which is correlated with oscillations of the condensate widths. The $z-y$ secondary mode coupling saturates after $\approx 22 \mathrm{~ms}$, signaling the end of the primary phase of rapid evolution. At this point the initial excess Cs single-particle energy concentrated along $z$ has been converted to $\mathrm{Yb}$ single-particle energy across both the $z$ and $y$ directions in comparable fractions. Smaller, secondary couplings along the $x$ direction (the weak axis of the trap) occur at longer timescales. Additionally, at longer times the abundant excitations in the system begin to thermalize, causing further damping and minimizing the amplitude of beating observed in the Cs CoM dynamics.

Earlier related studies of repulsively coupled nonlinear superfluids [9,32-34] have identified two distinct excitation mechanisms. Firstly, in a number-imbalanced system such as ours, the motion of the smaller superfluid through the larger component can lead to single-component excitations in the larger superfluid. For the attractive interaction in our system, this is analogous to stirring a single-component superfluid with a focused red-detuned laser beam [35,36]. Secondly, hydrodynamic considerations in the homogeneous limit reveal the existence of a dynamical instability whenever the relative motion of two superfluids exceeds some characteristic speed. In the limit of small interspecies interactions, this speed 

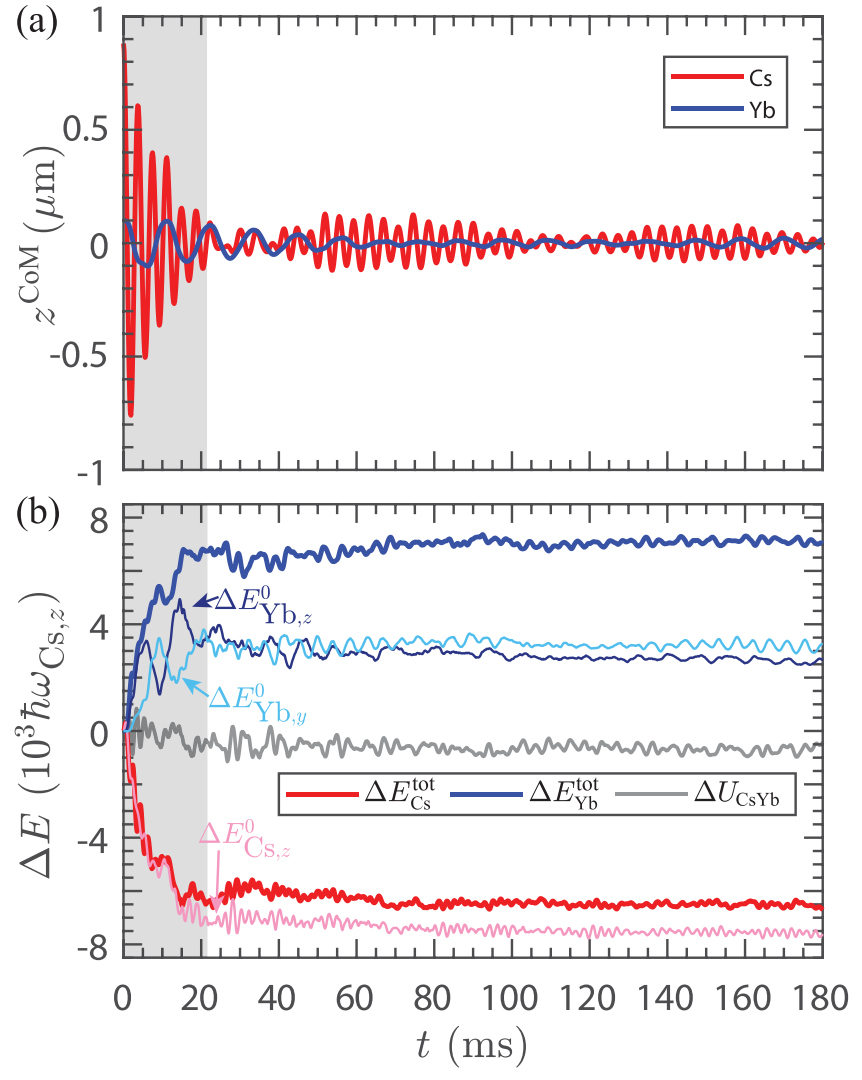

FIG. 2. Cs and Yb excitation and energy exchange calculated numerically in a coupled-GPE treatment with $\left(N_{\mathrm{Yb}}, N_{\mathrm{Cs}}\right)=(40,6.5) \times$ $10^{3}$. (a) Extended numerical evolution of the vertical CoM motions. (b) Changes in the total energy of $\mathrm{Cs}$ and $\mathrm{Yb}\left(\Delta E_{\mathrm{Cs} / \mathrm{Yb}}^{\mathrm{tot}}\right)$, the $\mathrm{Cs}-\mathrm{Yb}$ interaction energy $\left(\Delta U_{\mathrm{CsYb}}\right)$, and the main corresponding singleparticle energy along the $z$ and (for $\mathrm{Yb}$ only) the $y$ axis. Note the more gradual initial increase along $y$, consistent with a secondary excitation mechanism, and the initial out-of-phase oscillations between $z$ and $y$ directions for $\mathrm{Yb}$. The gray region shown in both (a) and (b) indicates the first $22 \mathrm{~ms}$ of evolution, where the primary damping and subsequent energy transfer occur.

approaches the sum of the sound velocities of the two (uncoupled) superfluids [4,32-34]. This counterflow instability leads to the simultaneous generation of pairs of excitations in both superfluids [32]. We believe that both these mechanisms are at play in our experiment as we show in Fig. 3.

In the left panel of Fig. 3(a) we plot the simulated CoM motion for $\mathrm{Cs}$ (red) and $\mathrm{Yb}$ (blue) over the first $22 \mathrm{~ms}$, where the dominant dynamics, energy transfer, and dissipation occur. The right panel zooms in further to examine the system behavior over the first Cs-oscillation cycle $\left(T_{\mathrm{Cs}} \approx 3.7 \mathrm{~ms}\right)$, which seeds the subsequent dynamics. We also track the CoM of the central bulge in the Yb BEC (dashed blue line), by limiting the CoM calculation to the central region where $\mathrm{Yb}$ is colocated with the Cs BEC $(|x|<10 \mu \mathrm{m},|y|<6 \mu \mathrm{m}$, and $|z|<3 \mu \mathrm{m})$. The contrast between the central $\mathrm{Yb}$ bulge and the total $\mathrm{Yb}$ CoM motion reveals evidence for secondary (noncentral) dynamics within $\mathrm{Yb}$. These secondary dynamics arise as a result of micromotions in $\mathrm{Yb}$ both within and beyond the region of $\mathrm{Cs}-\mathrm{Yb}$ overlap. Such micromotions are associated with the emerging excitations and include both in-phase and out-of-phase oscillations of the central bulge and the total $\mathrm{Yb}$ CoM.

The spatial-temporal evolution of the local velocity fields shown in Figs. 3(b) and 3(c) is useful for visualizing the two excitation regimes. The velocity field along the $z$ axis for $\mathrm{Cs}(\mathrm{Yb})$ is obtained from $v_{\mathrm{Cs}(\mathrm{Yb})}=$ $\hbar \nabla \arg \left[\psi_{\mathrm{Cs}(\mathrm{Yb})}(0,0, z)\right] / m_{\mathrm{Cs}(\mathrm{Yb})}$, where $m$ is the mass and $\psi$ is the condensate wave function. During the initial half oscillation $\left(0<t<T_{\mathrm{Cs}} / 2\right)$, Cs exhibits coherent harmonic motion, maintaining a constant phase profile across its vertical width. In contrast, during this same initial period, incoherent features gradually emerge in the $\mathrm{Yb}$ component in the form of counterpropagating density waves as the relative velocity between the two components increases, consistent with the single-excitation picture. Here, the Cs BEC both is smaller than $\mathrm{Yb}$ and executes faster oscillations. Therefore it acts as a perturbing attractive barrier, which travels through the $\mathrm{Yb} \mathrm{BEC}$ and drags along the central $\mathrm{Yb}$ density bulge. As shown in Fig. 3(c), the bulge begins to track the downward Cs motion at $t \sim 1 \mathrm{~ms}\left(T_{\mathrm{Cs}} / 4\right)$, which corresponds to the point of maximum downward Cs velocity. This in turn causes Cs to slow down as it continuously transfers energy to $\mathrm{Yb}$.

Counterflow dynamics occur when Cs reverses its motion at $t \approx 1.9 \mathrm{~ms}\left(T_{\mathrm{Cs}} / 2\right.$, dashed vertical line) while the central $\mathrm{Yb}$ bulge continues its downward motion. This creates a direct counterflow between the two components, whose magnitude increases over the next quarter cycle of the Cs oscillation (until $\left.t=3 T_{\mathrm{Cs}} / 4\right)$. The counterflow is clearly visible in Fig. 3(d), which depicts the spatial-temporal evolution of the scaled relative velocities $\left(v_{\mathrm{Cs}}-v_{\mathrm{Yb}}\right) /\left(c_{\mathrm{Cs}}+c_{\mathrm{Yb}}\right)$ along the $z$ axis. Here, the sum of the instantaneous local speeds of sound $\left(c_{\mathrm{Cs}}+c_{\mathrm{Yb}}\right)$ provides an upper bound for the critical velocity of the coupled system, with the onset of counterflow instabilities expected for $\left(v_{\mathrm{Cs}}-v_{\mathrm{Yb}}\right) /\left(c_{\mathrm{Cs}}+c_{\mathrm{Yb}}\right) \sim 1$. This choice is based on the predicted critical velocity of counterflow for two weakly coupled superfluids of $\left(c_{\mathrm{Cs}}+c_{\mathrm{Yb}}\right)$ [32,34], where $c_{i}=$ $\sqrt{g_{i} n_{i} / m_{i}}$ with $g_{i}=4 \pi \hbar^{2} a_{i i} / m_{i}$ is the critical sound speed for each individual component and the density $n_{i}$ is measured locally at each moment in time. The establishment of direct counterflow between the $\mathrm{Cs}$ and $\mathrm{Yb}$ components leads to the emergence of incoherent features in the propagation of $\mathrm{Cs}$ and coinciding secondary excitations of $\mathrm{Yb}$. These features are consistent with the generation of coupled excitations in the two components, as a result of the counterflow instability near the edges of the $\mathrm{Cs}-\mathrm{Yb}$ overlap region where the densities, and thus speeds of sound, are appropriately reduced. For reference, the edges of the Cs component $\left(n_{\mathrm{Cs}}=2 \mu \mathrm{m}^{-3}\right)$ are indicated in Figs. 3(c) and 3(d) by the pair of (approximately horizontal) black lines.

The moving Cs component also induces a secondary, at times opposing in direction, flow within the central $\mathrm{Yb}$ bulge, thus giving rise to the coexistence of flows in the same and opposing directions (the extension of comoving and countermoving modes to two superfluids which experience different trap frequencies [15]). The countermoving mode within the $\mathrm{Yb} \mathrm{BEC}$ is particularly evident at $t=T_{\mathrm{Cs}}$ (solid vertical line), when the Cs CoM next reverses its direction. Here, we see a stark difference in the behavior between the CoM motion of the central $\mathrm{Yb}$ bulge and that of the full $\mathrm{Yb}$ system [see 

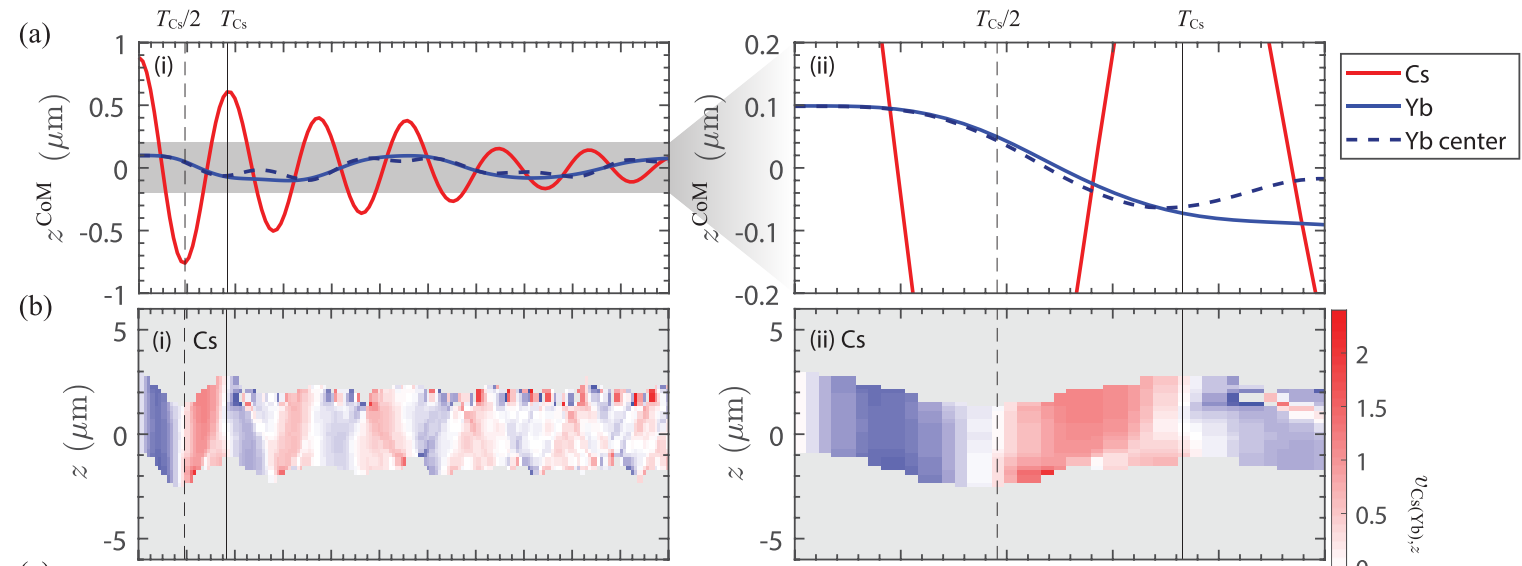

(c)
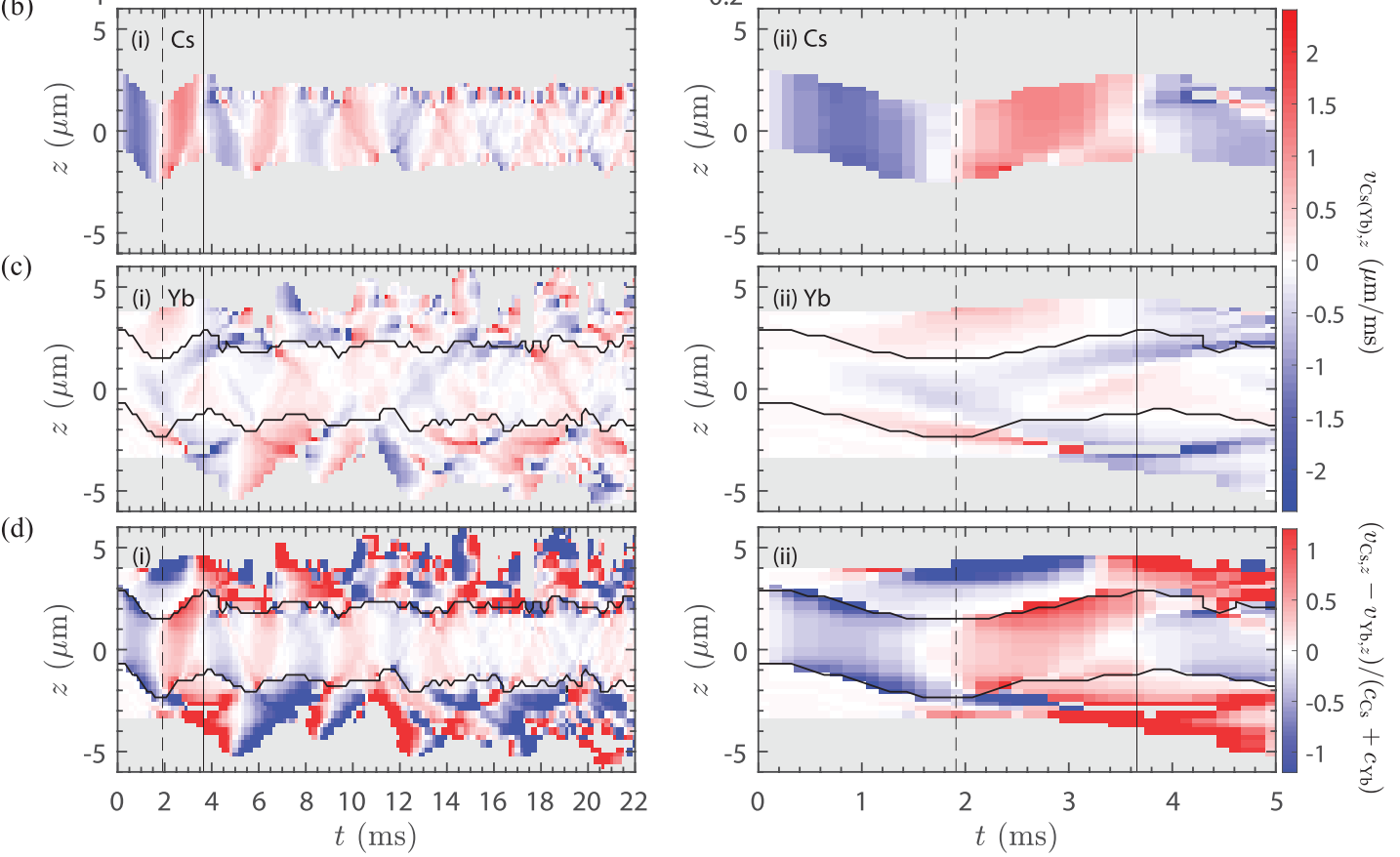

FIG. 3. Analysis of the Cs-Yb coupled dynamics during the first $22 \mathrm{~ms}$ [panels at left, (i)] and zoomed in to the first $5 \mathrm{~ms}$ [panels at right, (ii)]. All parameters are calculated from the numerical simulations used for Fig. 2. (a) Vertical CoM oscillation of Cs (red) and Yb (blue). The dashed blue line shows the CoM of the central "bulge" region of $\mathrm{Yb}$ (defined by $|x|<10 \mu \mathrm{m},|y|<6 \mu \mathrm{m}$, and $|z|<3 \mu \mathrm{m}$ ). (b) and (c) $\mathrm{Cs}$ and $\mathrm{Yb}$ velocity fields along the $z$ axis [defined by $\left.v_{\mathrm{Cs}(\mathrm{Yb})}=\hbar \nabla \arg \left[\psi_{\mathrm{Cs}(\mathrm{Yb})}(0,0, z)\right] / m_{\mathrm{Cs}(\mathrm{Yb})}\right]$. (d) Scaled relative local velocity $\left(v_{\mathrm{Cs}}-v_{\mathrm{Yb}}\right) /\left(c_{\mathrm{Cs}}+c_{\mathrm{Yb}}\right)$ along the $z$ axis, where $c_{\mathrm{Cs}(\mathrm{Yb})}=\sqrt{g_{\mathrm{Cs}(\mathrm{Yb})} n_{\mathrm{Cs}(\mathrm{Yb})} / m_{\mathrm{Cs}(\mathrm{Yb})}}$ is the local speed of sound evaluated using the local atom density. The velocity plots include a gray mask to filter out regions corresponding to Cs (b) and $\mathrm{Yb}[(\mathrm{c})$ and (d)] atomic densities lower than $2 \mu \mathrm{m}^{-3}$. The pairs of (approximately horizontal) black lines in (c) and (d) denote the vertical extent of the Cs velocity profile shown in (b). The dashed and solid vertical lines mark the Cs CoM turning points at $T_{\mathrm{Cs}} / 2 \approx 1.9 \mathrm{~ms}$ and $T_{\mathrm{Cs}} \approx 3.7 \mathrm{~ms}$, respectively.

panel (ii) of Fig. 3(a)]. The ensuing Yb motion, which extends beyond the region of $\mathrm{Cs}-\mathrm{Yb}$ overlap, feeds back into Cs by exciting phonons at the edges of the Cs component where its density is lowest [see Figs. 3(b)-3(d)], leading to a complicated energy transfer and phonon-excitation mechanism in both components. Such features can also be seen in the Fourier spectra shown in Fig. 4 revealing coexistence of dynamical components for both $\mathrm{Cs}$ and $\mathrm{Yb}$ across the same dominant frequencies. We have further verified that smaller initial amplitude oscillations give rise to reduced damping and fewer excitations.

\section{Beating and long-time dynamics}

Although the dominant dynamics occur during the experimentally probed timescale of $22 \mathrm{~ms}$, closer inspection of the long-time coupled evolution for a fixed total atom number reveals smaller-scale energy transfers between the $\mathrm{Cs}$ and $\mathrm{Yb}$ components. This results in partial revivals in the Cs CoM oscillations, as shown in Fig. 2(a). Such beating arises from the existence of two closely located dominant frequencies for
Cs (both within a few percent of the natural Cs frequency), the origin of which can be attributed to the coexisting secondary dynamical micromotions (combinations of appropriate comoving and countermoving modes) in the central $z$ region. This is in contrast to the beating observed in Ref. [9], which resulted from the two components having comparable trap frequencies.

Here, we use our coupled-GPE simulations to shed more light on the $\mathrm{Cs}-\mathrm{Yb}$ coupled dynamics, by analyzing the mixture's excitation spectrum, through the Fourier spectra of $z_{\mathrm{Cs}(\mathrm{Yb})}^{\mathrm{CoM}}$. As shown in Fig. 4, we observe a multitude of excitation frequencies in both components, consistent with the complicated excitation generation discussed above. The Fourier spectra reveal the simultaneous presence of dominant oscillation modes for both $\mathrm{Cs}$ and $\mathrm{Yb}$, both close to the natural frequencies of each component and close to the difference (and average value) of such frequencies, indicating the strong presence of comoving and countermoving oscillating components.

As expected, the dominant dynamics for each component arise at frequencies close to their natural frequency, slightly 


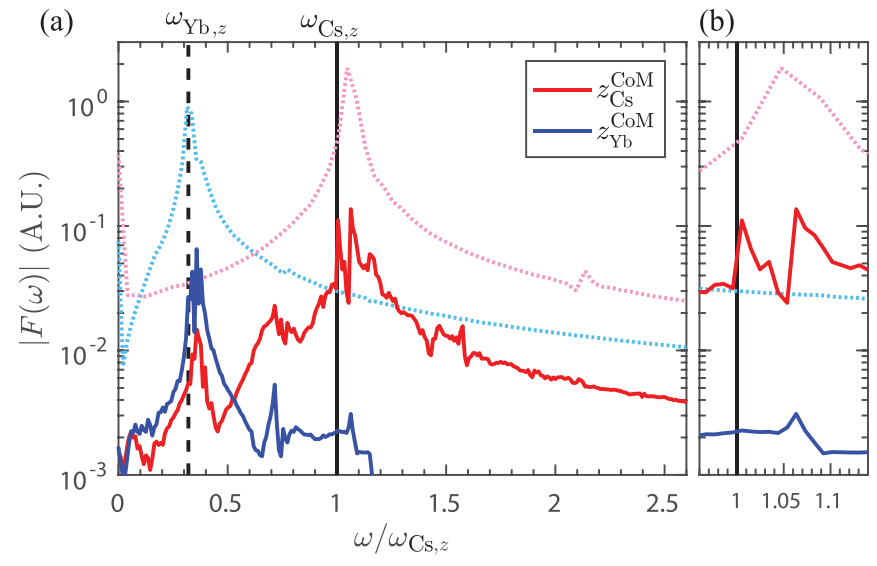

FIG. 4. The Fourier spectra of $z_{\mathrm{Cs}(\mathrm{Yb})}^{\mathrm{CoM}}$ for $\left(N_{\mathrm{Yb}}, N_{\mathrm{Cs}}\right)=$ $(40,6.5) \times 10^{3}$ (based on an evolution of $413.8 \mathrm{~ms}$ giving a spectral resolution of $\left.\omega_{\mathrm{Cs}, z} / 650\right)$. The red and blue solid lines are the spectra of $\mathrm{Cs}$ and $\mathrm{Yb}$, respectively. The pink and blue dotted lines are the corresponding spectra for the case where the other component is static (see text for details). In (b) we zoom in to the region around $\omega / \omega_{\mathrm{Cs}, z}=1$ to show the splitting of the oscillation frequency in Cs.

upshifted due to the mutual attractive mean-field potential. To better characterize the role of the attractive mean-field potential, we have performed corresponding GPE simulations for $\mathrm{Cs}(\mathrm{Yb})$ when coupled to a static mean-field contribution from the second component [i.e., $\mathrm{Yb}(\mathrm{Cs})]$. At each time step the static component is reset to its configuration at $t=0$, thereby only contributing to the effective (trap + mean field) potential felt by the main component. The resulting spectra are shown for $\mathrm{Cs}(\mathrm{Yb})$ by the pink (light blue) dotted line, clearly revealing the previously mentioned $\approx 5 \%$ frequency upshift for $\mathrm{Cs}$. The more massive $\mathrm{Yb}$ component, which is affected less by the Cs attraction, experiences a smaller upshift.

Returning to the coupled dynamics, the spectra clearly demonstrate splitting of the dominant upshifted frequency of each component into multiple frequencies. Such frequencies are generally found to be upshifted from the natural frequency and to lie on either side of the static-mean-field contribution, thus highlighting the dynamical role of the other component. For Cs, we find two dominant frequencies, of broadly comparable importance. For the "primary" atom number combination considered here, these are $\omega_{\mathrm{Cs}, z}^{(1)} \sim 1.063 \omega_{\mathrm{Cs}, z}$ and $\omega_{\mathrm{Cs}, z}^{(2)} \sim 1.005 \omega_{\mathrm{Cs}, z}$. The difference in these two frequencies establishes a timescale $2 \pi /|\Delta \omega| \approx 69 \mathrm{~ms}$ which leads to the underlying beating cycle reported in Fig. 2(a). The period of this beating cycle is highly sensitive to the particular atom numbers considered (see Appendix B 2). This sensitivity, combined with rapid damping, makes it hard to characterize such effects in our current experiments.

\section{MIXTURE COLLAPSE}

In a separate study, we explore the dynamical instabilities of the mixture further by tuning the relative mean-field contributions through varying the Cs scattering length. The attractive interspecies interaction between $\mathrm{Cs}$ and ${ }^{174} \mathrm{Yb}$ gives rise to mean-field collapse of the Cs BEC mediated by
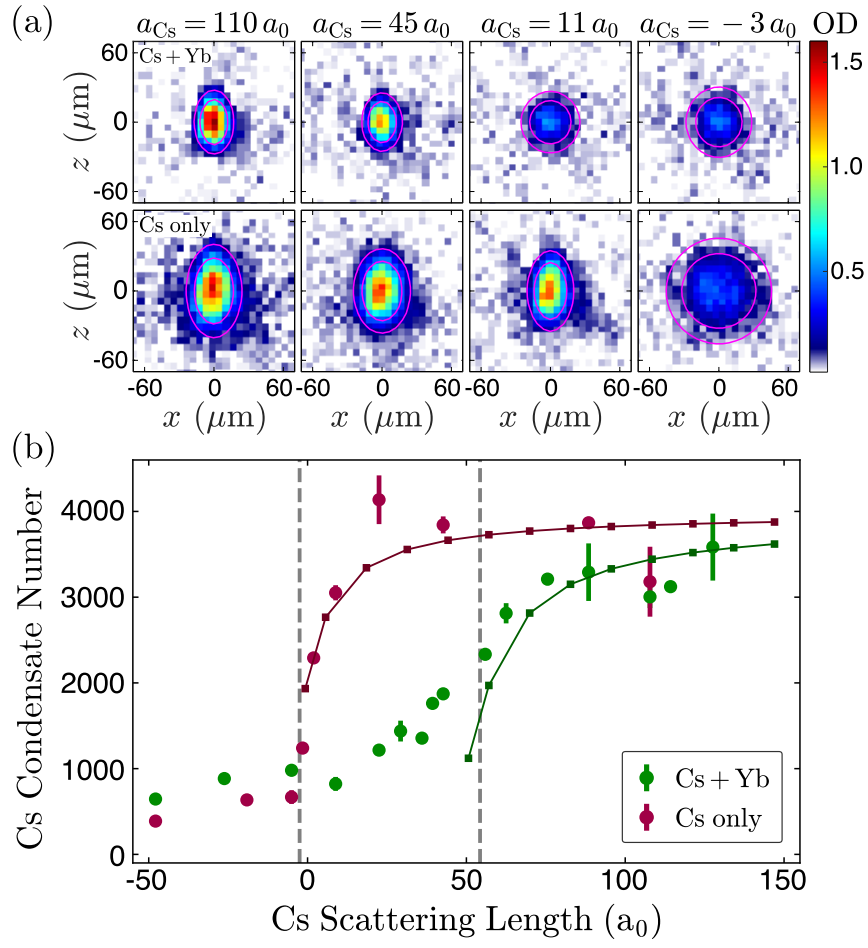

FIG. 5. Dual-species collapse. (a) Cs collapse mediated by ${ }^{174} \mathrm{Yb}$ (top row) and Cs single-species collapse (bottom row). Images show the Cs BEC optical depth (OD) profile for varying $a_{\mathrm{Cs}}$. Pink contours show $1 / e$ and $1 / e^{2}$ of peak OD. (b) Cs condensate number as a function of Cs scattering length for Cs collapse mediated by ${ }^{174} \mathrm{Yb}$ (green circles) and Cs single-species collapse (red circles). The dashed line at $a_{\mathrm{Cs}}=-2.5 a_{0}$ denotes the theoretically predicted single-species collapse threshold for $N_{\mathrm{Cs}}=4 \times 10^{3}$. The dashed line at $a_{\mathrm{Cs}}=54.3 a_{0}$ corresponds to the cancellation of mean-field interactions $\delta g=0$. The results of GPE simulations with three-body loss are shown with green $(\mathrm{Cs}+\mathrm{Yb})$ and red squares (Cs only); lines are guides to the eye.

the presence of the $\mathrm{Yb}$ BEC, as shown in Fig. 5. In both single-component and dual-species BECs, the onset of collapse is related to a mechanical instability arising from the population of imaginary Bogoliubov modes [37]. In a singlecomponent BEC, collapse [38-42], along with its associated density-dependent atom loss, occurs when the intraspecies interaction becomes sufficiently attractive to overcome the zero-point kinetic energy associated with the harmonic trapping potential. For a given atom number $N$, the scattering length $a^{\text {(crit) }}$ associated with the onset of collapse is given by $\left|a^{\text {(crit) }}\right|=C \sqrt{\hbar /(m \bar{\omega})} / N$, where $\bar{\omega}$ is the geometric mean of the trap frequencies and $C$ is a numerical constant weakly dependent on the trap geometry $[40,43,44]$. In contrast, the dual-species collapse threshold primarily depends on the balance of the inter- and intraspecies interactions, and the critical point for collapse is determined by the parameter $\delta g=g_{12}+$ $\sqrt{g_{11} g_{22}}$, which describes the balance of mean-field interactions in the system. Here, $g_{i j}=2 \pi \hbar^{2} a_{i j}\left(m_{i}+m_{j}\right) /\left(m_{i} m_{j}\right)$ is the interaction coupling constant. When $\delta g<0$, the modes of the density branch of the Bogoliubov excitation spectrum become imaginary, leading to the onset of mechanical instabilities and subsequent collapse [16-18]. 
In our experiment, we approach the collapse instability by varying $a_{\mathrm{Cs}}$ via a magnetic Feshbach resonance. We begin with a dual-species BEC formed with $a_{\mathrm{Cs}}=147 a_{0}$, with atom numbers $\left(N_{\mathrm{Yb}}, N_{\mathrm{Cs}}\right)=(50,4) \times 10^{3}$. We ramp $a_{\mathrm{Cs}}$ to the desired value over $10 \mathrm{~ms}$, hold for $30 \mathrm{~ms}$, and then ramp back to $a_{\mathrm{Cs}}=147 a_{0}$ over $10 \mathrm{~ms}$ prior to ToF expansion and imaging. As shown in Fig. 5, we observe the onset of collapse as a marked decrease in Cs atom number due to density-dependent three-body losses $\left(K_{3, \mathrm{Cs}} \sim 1 \times 10^{-27}\right.$ $\mathrm{cm}^{6} / \mathrm{s}[28,29,45]$ for the range of magnetic fields explored). Figure 5(a) shows absorption images of the Cs BECs for varying $C s$ scattering lengths, both with (top row) and without (bottom row) $\mathrm{Yb}$ present. The presence of $\mathrm{Yb}$ causes the collapse instability to occur at $a_{\mathrm{Cs}}>0$, where we would expect a stable single-component Cs BEC. In addition to atom loss, we also observe a change in aspect ratio for the Cs cloud. We quantify the collapse in Fig. 5(b) by plotting Cs condensate number versus Cs scattering length. Red circles denote Cs single-species collapse occurring when $a_{\mathrm{Cs}} \leqslant 0$. The vertical line at $a_{\mathrm{Cs}}=-2.5 a_{0}$ marks the predicted collapse point $a_{\mathrm{Cs}}^{\text {(crit) }}$ for $N_{\mathrm{Cs}}=4 \times 10^{3}$ atoms. Green circles show Cs collapse mediated by the presence of $\mathrm{Yb}$. The vertical line at $a_{\mathrm{Cs}}=$ $54.3 a_{0}$ marks the point where $\delta g=0$ for the $\mathrm{Cs}-\mathrm{Yb}$ mixture.

We model the experimental protocol numerically using the 3D coupled GPEs with a density-dependent three-body loss term for Cs and $\omega_{\mathrm{Cs}, z}=2 \pi \times 260 \mathrm{~Hz}$ (see Appendixes A and $\mathrm{C}$ ). The simulation results are shown in Fig. 5(b) for $\mathrm{Cs}+\mathrm{Yb}$ (green squares) and Cs only (red squares). For Cs scattering lengths lower than the range shown, the simulations do not converge at the highest resolutions we could feasibly use, indicative of mean-field collapse [31]. We find good agreement with the experimental results using a thermal Cs three-body loss coefficient of $K_{3, \mathrm{Cs}}=1.8 \times 10^{-27}$ $\mathrm{cm}^{6} / \mathrm{s}$. This is in reasonable agreement with other experimental measurements of $K_{3, \text { Cs }}[28,29,45]$ (see Appendix C 2). We observe that the Yb-mediated collapse is broader than predicted by the GPE simulations, while the Cs-only collapse is not. Because both types of collapse were observed at a similar temperature, finite-temperature effects do not appear to offer a straightforward explanation for this broadening. This raises the possibility that the broadening may be indicative of beyond-mean-field effects, related to the formation of quantum droplets recently observed in both $\mathrm{K}$ spin mixtures $[19,20]$ and $\mathrm{Rb}-\mathrm{K}$ mixtures [21]. For $\mathrm{Cs}+\mathrm{Yb}$, the weak and attractive net mean-field interaction $\delta g$ required to support the droplet phase arises for Cs scattering lengths below and in the vicinity of $a_{\mathrm{Cs}}=54.3 a_{0}$. This possibility is an interesting direction for future studies.

\section{CONCLUSIONS}

We have probed the collective dynamics of a quantum degenerate Bose-Bose mixture of $\mathrm{Cs}$ and ${ }^{174} \mathrm{Yb}$ with attractive interspecies interactions. Observations of the CoM oscillations of $\mathrm{Cs}$ in the presence of $\mathrm{Yb}$ have revealed a measurable increase in the dipole mode frequency and significant damping as energy is transferred to $\mathrm{Yb}$. We find good agreement with numerical simulations which highlight the complexity of the coupled dynamics. We have also investigated the stability of the degenerate mixture by crossing the transition for dual-species collapse and find good agreement with coupledGPE simulations that include a Cs three-body loss term. This opens up new prospects for the study of the BEC-to-quantumdroplet phase transition.

In this context, a key advantage of Cs-Yb mixtures is that the Cs scattering length may be precisely tuned without affecting $\mathrm{Yb}$. Additionally, the very different atomic structures of $\mathrm{Cs}$ and $\mathrm{Yb}$ enable species-specific optical traps with low photon scattering rates. These advantages may prove invaluable for precise studies of binary fluid dynamics in both Bose-Bose and Bose-Fermi Cs- $\mathrm{Yb}$ mixtures.

The data presented in this paper are available [46].

\section{ACKNOWLEDGMENTS}

We acknowledge support from the UK Engineering and Physical Sciences Research Council (Grants No. EP/P01058X/1, No. EP/T015241/1, and No. $\mathrm{EP} / \mathrm{T} 01573 \mathrm{X} / 1$ ). I-K.L. and N.P.P. acknowledge funding from the Quantera ERA-NET cofund project NAQUAS (EPSRC Grant No. EP/R043434/1) and the support of the Nvidia Corporation through the donation of the Tesla K40 graphics processing unit used for the collective mode dynamics.

\section{APPENDIX A: THEORETICAL MODEL}

Our theoretical model is based on a three-dimensional mean-field description by the coupled Gross-Pitaevskii equations (GPEs)

$$
\begin{aligned}
i \hbar \frac{\partial \psi_{\mathrm{Yb}}}{\partial t}= & {\left[-\frac{\hbar^{2} \nabla^{2}}{2 m_{\mathrm{Yb}}}+V_{\mathrm{Yb}}(\mathbf{r})+g_{\mathrm{Yb}}\left|\psi_{\mathrm{Yb}}\right|^{2}\right.} \\
& \left.+g_{\mathrm{CSYb}}\left|\psi_{\mathrm{Cs}}\right|^{2}-\mu_{\mathrm{Yb}}\right] \psi_{\mathrm{Yb}}
\end{aligned}
$$

and

$$
\begin{aligned}
i \hbar \frac{\partial \psi_{\mathrm{Cs}}}{\partial t}= & {\left[-\frac{\hbar^{2} \nabla^{2}}{2 m_{\mathrm{Cs}}}+V_{\mathrm{Cs}}(\mathbf{r})+g_{\mathrm{Cs}}\left|\psi_{\mathrm{Cs}}\right|^{2}\right.} \\
& \left.+g_{\mathrm{Cs} \mathrm{Yb}}\left|\psi_{\mathrm{Yb}}\right|^{2}-\mu_{\mathrm{Cs}}-\frac{i \hbar K_{3, \mathrm{Cs}}^{(\mathrm{C})}}{2}\right] \psi_{\mathrm{Cs}},
\end{aligned}
$$

where $\psi_{\mathrm{Yb}(\mathrm{Cs})}$ is the mean-field wave function of $\mathrm{Yb}(\mathrm{Cs})$ trapped in the harmonic potential $V_{\mathrm{Yb}(\mathrm{Cs})}(\mathbf{r})$ and $g_{i j}=$ $2 \pi \hbar^{2} a_{i j}\left(m_{i}+m_{j}\right) /\left(m_{i} m_{j}\right)$ is the interaction coupling constant. The Cs three-body loss coefficient for the condensate is $K_{3, \mathrm{Cs}}^{(\mathrm{C})}$, while the corresponding thermal coefficient $K_{3, \mathrm{Cs}}$ is a factor of 3 ! higher [47]. The wave functions are normalized to the total atom numbers,

$$
\int d \mathbf{r}\left|\psi_{\mathrm{Yb}}\right|^{2}=N_{\mathrm{Yb}} \quad \text { and } \quad \int d \mathbf{r}\left|\psi_{\mathrm{Cs}}\right|^{2}=N_{\mathrm{Cs}} .
$$

The trapping potentials are considered as

$$
V_{\mathrm{Yb}}=\frac{m_{\mathrm{Yb}}}{2}\left(\omega_{\mathrm{Yb}, x}^{2} x^{2}+\omega_{\mathrm{Yb}, y}^{2} y^{2}+\omega_{\mathrm{Yb}, z}^{2} z^{2}\right)
$$

and

$$
V_{\mathrm{Cs}}=\frac{m_{\mathrm{Cs}}}{2}\left[\omega_{\mathrm{Cs}, \tilde{x}}^{2} \tilde{x}^{2}+\omega_{\mathrm{Cs}, \tilde{y}}^{2} \tilde{y}^{2}+\omega_{\mathrm{Cs}, z}^{2}(z-\Delta z)^{2}\right] .
$$


Here, the $\tilde{x}$ and $\tilde{y}$ axes in $V_{\mathrm{Cs}}$ indicate that the weak trap axis $(\tilde{x})$ and one of the transverse trap axes $(\tilde{y})$ of Cs are rotated by $\alpha=$ $20^{\circ}$ with respect to the $x$ (weak) and $y$ axes of $\mathrm{Yb}$, while the $z$ axis remains coincided, in agreement with the experimental setup. $\Delta z$ is the displacement of the Cs trap minimum from the minimum of the $\mathrm{Yb}$ trap.

The coupled GPEs are simulated using Fourier pseudospectral methods. In the simulations, the trapping frequencies are as described in the relevant sections, and we take $a_{\mathrm{Yb}}=105 a_{0}$ and $a_{\mathrm{CsYb}}=-75 a_{0}$, where $a_{0}$ is the Bohr radius. In Appendix B we define parameters useful for comparison with the experimental measurements and for the analysis of the dynamics discussed in the main text.

\section{APPENDIX B: COUPLED OSCILLATIONS}

The coupled oscillations of $\mathrm{Cs}$ and $\mathrm{Yb}$ BECs triggered by the sudden removal of the Cs trap displacement are modeled by the GPEs without the inclusion of the three-body loss term (setting $K_{3, \mathrm{Cs}}^{(\mathrm{C})}=0$ ), based on an initial Cs trap displacement $\Delta z=0.95 \mu \mathrm{m}, \omega_{\mathrm{Cs}, z}=2 \pi \times 250 \mathrm{~Hz}$, and fixed $a_{\mathrm{Cs}}=$ $275 a_{0}$. The initial Cs trap displacement is implemented in the stationary initial condition, generated by solving Eq. (A2) with the imaginary-time propagation method. Subsequently, $\Delta z$ is set to zero for all $t \geqslant 0$ to mimic the experimental procedure. The required set of particle numbers for the initial condition is reached by a self-consistent choice of the chemical potentials $\mu_{\mathrm{Cs}(\mathrm{Yb})}$ [48] during imaginary-time evolution.

The time propagation is conducted by the Runge-Kutta fourth-order method with a fixed time step $d t=10^{-3} / \omega_{\mathrm{Cs}, z} \approx$ $6.36 \times 10^{-4} \mathrm{~ms}$ and a grid discretization of $\Delta x=\Delta y=$ $\Delta z=0.5 l_{z}$ with $l_{z}=\sqrt{\hbar / m_{\mathrm{Cs}} \omega_{\mathrm{Cs}, z}} \approx 0.55 \mu \mathrm{m}$ found to be sufficient to perform the coupled oscillation dynamics (conserving both the particle number and total energy during the whole evolution). The simulation box size is set to be at least 1.5 of the largest noncoupled Thomas-Fermi radii of the two clouds along each direction to ensure that the dynamics are not affected by the box size (which, for the case discussed in the main text, corresponds to $\left.66.2 \times 19.8 \times 16.5 \mu \mathrm{m}^{3}\right)$. The numerical simulations are implemented using MATLAB 2018B on an Nvidia Tesla K40 graphics processing unit (GPU) card.

Our numerical simulations focus on the characterization of the in situ center of mass (CoM) along the $z$ direction

$$
z_{\mathrm{Cs}(\mathrm{Yb})}^{\mathrm{CM}}=\frac{1}{N_{\mathrm{Cs}(\mathrm{Yb})}} \int d \mathbf{r} z\left|\psi_{\mathrm{Cs}(\mathrm{Yb})}(\mathbf{r})\right|^{2},
$$

for each species. A full time-of-flight (ToF) simulation is numerically forbidden due to the huge computational size constraints imposed by the $V_{\mathrm{Cs}(\mathrm{Yb})}$ anisotropy. Instead, direct comparison with the experimental ToF data is facilitated by mapping the numerical in situ results to a ToF measurement by

$$
\text { Cs ToF position } \approx \tau_{\mathrm{ToF}} \frac{d z_{\mathrm{Cs}}^{\mathrm{CoM}}(t)}{d t},
$$

using the experimental ToF expansion time $\tau_{\mathrm{ToF}}=40 \mathrm{~ms}$ and assuming that the condensate carries its moving velocity during the ToF expansion. The time derivative is computed by the central-finite-difference method, namely,

$$
\frac{d z_{\mathrm{Cs}}^{\mathrm{CoM}}(t)}{d t} \approx \frac{\left[z_{\mathrm{Cs}}^{\mathrm{CoM}}(t+0.5 \Delta t)-z_{\mathrm{Cs}}^{\mathrm{CoM}}(t-0.5 \Delta t)\right]}{\Delta t},
$$

with $\Delta t=0.5 / \omega_{\mathrm{Cs}, z}$. This mapping provides an excellent agreement of numerical predictions with experimental observations as shown in Fig. 1(d).

Energy calculations across the three directions $x, y$, and $z$ (collectively labeled by $v$ ) are based on the single-particle Hamiltonian contributions

$$
E_{\mathrm{Cs}(\mathrm{Yb}), v}^{0}=\int d \mathbf{r} \psi_{\mathrm{Cs}(\mathrm{Yb})}^{*} \hat{H}_{\mathrm{Cs}(\mathrm{Yb}), v}^{0} \psi_{\mathrm{Cs}(\mathrm{Yb})},
$$

where

$$
\begin{aligned}
\hat{H}_{\mathrm{Cs}(\mathrm{Yb}), v}^{0} & =\mathrm{KE}_{\mathrm{Cs}(\mathrm{Yb}), v}+V_{\mathrm{Cs}(\mathrm{Yb}), v} \\
& =-\frac{\hbar^{2} \partial_{v}^{2}}{2 m_{\mathrm{Cs}(\mathrm{Yb})}}+\frac{m_{\mathrm{Cs}(\mathrm{Yb})}}{2} \omega_{\mathrm{Cs}(\mathrm{Yb}), v}^{2} v^{2}
\end{aligned}
$$

includes both kinetic energy (KE) and potential energy $(V)$ contributions. The intra- and interspecies interaction energies are evaluated by

$$
U_{\mathrm{Cs}(\mathrm{Yb})}=\frac{g_{\mathrm{Cs}(\mathrm{Yb})}}{2} \int d \mathbf{r}\left|\psi_{\mathrm{Cs}(\mathrm{Yb})}\right|^{4}
$$

and

$$
U_{\mathrm{CsYb}}=g_{\mathrm{CsYb}} \int d \mathbf{r}\left|\psi_{\mathrm{Cs}}\right|^{2}\left|\psi_{\mathrm{Yb}}\right|^{2},
$$

respectively.

\section{Mean-field frequency shift}

The frequency shift of dynamical collective modes of coupled superfluids due to the cross interaction between components has been studied in the context of both Bose-Bose and Bose-Fermi superfluid mixtures [9,34,49-51]. In the $\mathrm{Cs}-\mathrm{Yb}$ mixture under consideration here, the Cs particle number is much smaller than the $\mathrm{Yb}$ number. We can therefore neglect the back action of $\mathrm{Cs}$ on $\mathrm{Yb}$. As a consequence, the $\mathrm{Yb}$ component oscillates with the harmonic trap frequency $\omega_{\mathrm{Yb}, z}$. Moreover, within the Thomas-Fermi approximation, the $\mathrm{Yb}$ density profile, given by $n_{\mathrm{Yb}}(z)=\left[\mu_{\mathrm{Yb}}-V_{\mathrm{Yb}, z}(z)\right] / g_{\mathrm{Yb}}$, can be used to estimate the effective potential acting on Cs due to interspecies interactions. The effective Cs potential along the $z$ axis is then $V_{\mathrm{Cs}, z}^{\mathrm{eff}}(z) \approx V_{\mathrm{Cs}, z}+g_{\mathrm{CsYb}} n_{\mathrm{Yb}}(z)$. This potential has an effective harmonic frequency of

$$
\omega_{\mathrm{Cs}, z}^{\mathrm{eff}}=\omega_{\mathrm{Cs}, z} \sqrt{1-\frac{a_{\mathrm{Cs} Y \mathrm{~b}} m_{\mathrm{Yb}}^{2}}{a_{\mathrm{Yb}} M_{\mu} m_{\mathrm{Cs}}} \frac{\omega_{\mathrm{Yb}, z}^{2}}{\omega_{\mathrm{Cs}, z}^{2}}},
$$

where $M_{\mu}=2 m_{\mathrm{Yb}} m_{\mathrm{Cs}} /\left(m_{\mathrm{Yb}}+m_{\mathrm{Cs}}\right)$ is the reduced mass. This simple formula predicts $\omega_{\mathrm{Cs}, z}^{\mathrm{eff}} \approx 1.05 \omega_{\mathrm{Cs}, z}$, which captures the effective frequency for the Cs BEC in the presence of a static Yb BEC.

\section{Dependence on particle number and axis rotation}

All the results presented in this section are based on a definite particle number combination, with the finer details of the dynamics highly dependent on the relative $\mathrm{Cs} / \mathrm{Yb}$ atom number. Figure 6 compares the experimental data (green 


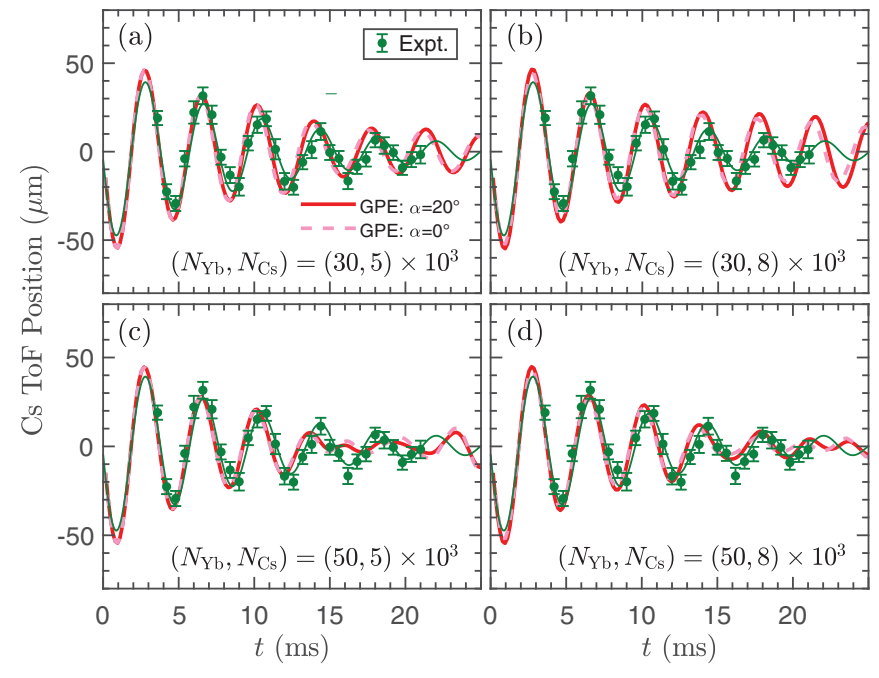

FIG. 6. (a)-(d) ToF Cs CoM positions along the $z$ direction in the presence of the $\mathrm{Yb}$ component predicted numerically (red line) and measured experimentally (green circles). The experimental data set is compared with simulations corresponding to different atom numbers, consistent with atom number variations of up to $1.25 \sigma$ from the "primary" values $\left(N_{\mathrm{Yb}}, N_{\mathrm{Cs}}\right)=(40,6.5) \times 10^{3}$. In addition to numerical predictions in the actual experimental geometry with $\alpha=20^{\circ}$ (solid red lines) (as also presented in the main text), the corresponding dynamics for $\alpha=0^{\circ}$ are also shown (dashed pink lines). The green line shown is the corresponding best fit to the experimental data set.

circles/line) against numerical simulations (red line) based on four different (definite) atom number combinations, chosen here to reflect number variations of up to $\approx 1.25 \sigma$ from the "primary" atom number combination used in the main text, $N_{\mathrm{Cs}}=(6.5 \pm 1.5) \times 10^{3}$ and $N_{\mathrm{Yb}}=(40 \pm 10) \times 10^{3}$. Here, $\sigma \sim 20 \%$ is the standard deviation in the experimental atom numbers. These reveal good overall agreement over the range of available experimental data, but with a sensitivity of the late-time $[t>(15-20) \mathrm{ms}]$ oscillations to the precise atom numbers (and, in particular, to the $\mathrm{Yb}$ atom number, which is the largest component). With this in mind, we focus our analysis in this paper on the primary atom number combination of $\left(N_{\mathrm{Yb}}, N_{\mathrm{Cs}}\right)=(40,6.5) \times 10^{3}$, which is in broad agreement with the values measured experimentally and leads to excellent agreement with the experimental observations shown in Fig. 1(c) of the main text. [Recall that Fig. 1(c) of the main text is based on weighted combinations of different atom numbers, statistically distributed around the primary mean value].

We also investigated the effect of the rotation of the Cs trapping potential from that of the $\mathrm{Yb}$ trap. Specifically, the simulated dynamics with a rotation angle of $\alpha=20^{\circ}$ (solid red lines in Fig. 6) are contrasted with corresponding dynamics in the absence of any rotation $\left(\alpha=0^{\circ}\right.$, dashed pink lines). The near-perfect overlap over the probed timescales conclusively rules out mismatch of the axes as a primary reason for the observed dynamics.

\section{Density snapshots}

To better visualize the complicated dynamics inherent in the coupled evolution of our system, we provide representa- tive snapshots of the $\mathrm{Cs}$ and $\mathrm{Yb}$ density profiles in Fig. 7. Here, we show 3D density isosurfaces and their corresponding $1 \mathrm{D}$ and $2 \mathrm{D}$ sliced density profiles during the first $\approx 22 \mathrm{~ms}$ of coupled evolution. A video of the full evolution, which also includes later times, can be found in the Supplemental Material [31]. From these, we can see the different stages of the evolution, including the $\mathrm{Yb}$ component being dragged by Cs over the first $\approx 2 \mathrm{~ms}$, the subsequent double-peak distribution of $\mathrm{Yb}$ along the $z$ axis, and the gradual excitations along different directions, with excitations along $x$ evident by $\approx 21 \mathrm{~ms}$.

\section{APPENDIX C: COLLAPSE PROPERTIES AND DYNAMICS}

We model the experiment using a three-dimensional mean-field description, expressed mathematically as twocomponent coupled GPEs with an added three-body loss term [52] for Cs as detailed in Eqs. (A1)-(A5). Here, we have $\Delta z=0, \omega_{\mathrm{Cs}, z}=2 \pi \times 260 \mathrm{~Hz}$, and a time-varying $a_{\mathrm{Cs}}$. Our numerical simulations of these coupled GPEs, described below, use Fourier pseudospectral methods with adaptive Runge-Kutta time stepping and are implemented using the XMDS2 software [53].

\section{Ground states and static collapse analysis}

One can attempt to find ground-state solutions $\left[\partial_{t} \psi_{\mathrm{Yb}}=\right.$ $\left.\partial_{t} \psi_{\mathrm{Cs}}=0\right]$ to the coupled GPEs with a fixed Cs scattering length and zero three-body loss $K_{3, \mathrm{Cs}}^{(\mathrm{C})}=0$. Below a critical value of Cs scattering length, $a_{\mathrm{Cs}}^{\text {(crit) }}$, collapse occurs [41], and mean-field ground-state solutions cease to exist. We use imaginary-time propagation to find ground-state solutions numerically. For Cs only, with $N_{\mathrm{Cs}}=4 \times 10^{3}$, we find $a_{\mathrm{Cs}}^{\text {(crit) }} \approx$ $-2.15 a_{0}$. We found that an approximate variational calculation [54,55] assuming a Gaussian ground-state profile (and ignoring rotation of trap axes) yields a fairly similar result $\left(a_{\mathrm{Cs}}^{\text {(crit })} \approx-2.48 a_{0}\right.$ ) without the computational effort of solving the GPE. With $\mathrm{Yb}$ also present, with atom numbers $N_{\mathrm{Cs}}=$ $4 \times 10^{3}$ and $N_{\mathrm{Yb}}=5 \times 10^{4}$, we find $a_{\mathrm{Cs}}^{\text {(crit) }} \approx 51.25 a_{0}$ using imaginary-time propagation. In this case, a Gaussian variational calculation gives incorrect results $\left(a_{\mathrm{Cs}}^{\text {(crit) }} \approx-1.75 a_{0}\right)$ since the $\mathrm{Yb}$ condensate is far from having a Gaussian profile.

\section{Dynamical collapse analysis}

To model the dynamic collapse experiments reported in the main text, we perform real-time simulations of the coupled GPEs that are intended to match the experimental protocol. We begin from the $\mathrm{Cs}+\mathrm{Yb}$ ground-state solution, found as described above, with $a_{\mathrm{Cs}}(t=0)=147 a_{0}, N_{\mathrm{Cs}}=4 \times 10^{3}$, and $N_{\mathrm{Yb}}=5 \times 10^{4}$. When simulating the Cs-only dynamic collapse experiments, we continue to find the $\mathrm{Cs}+\mathrm{Yb}$ ground state, but we remove $\mathrm{Yb}$ atoms immediately at time $t=0$ by setting $\psi_{\mathrm{Yb}}=0$, in analogy to the experimental protocol.

We then perform a real-time simulation of the coupled GPEs in which the Cs three-body loss coefficient is set to a constant, nonzero, value and the Cs scattering length is varied in time to match the experimental protocol. Specifically, we implement in sequence a hold at $a_{\mathrm{Cs}}(t)=147 a_{0}$ for $5 \mathrm{~ms}$, a 10-ms linear ramp to $a_{\mathrm{Cs}}(t)=a_{\mathrm{Cs}}^{\text {(jump) }}$, a hold at this value for 

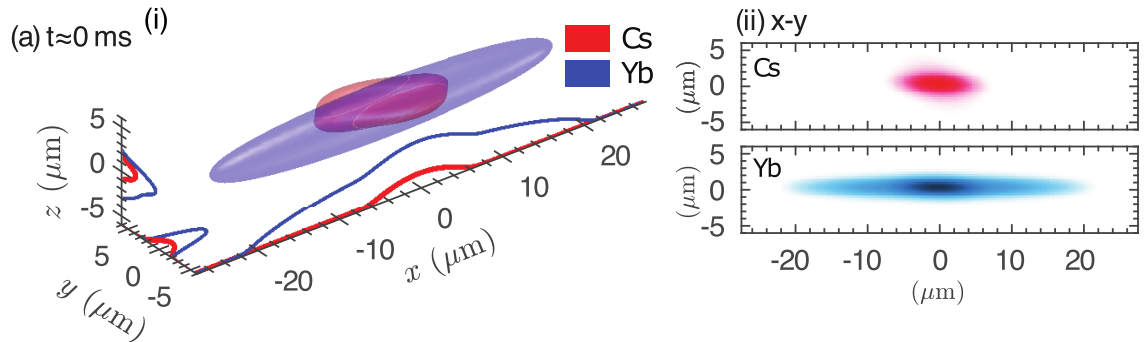

(iii) $\mathrm{x}-\mathrm{z}$

(iv) $y-z$

(b) $\mathrm{t} \approx 1.91 \mathrm{~ms}$
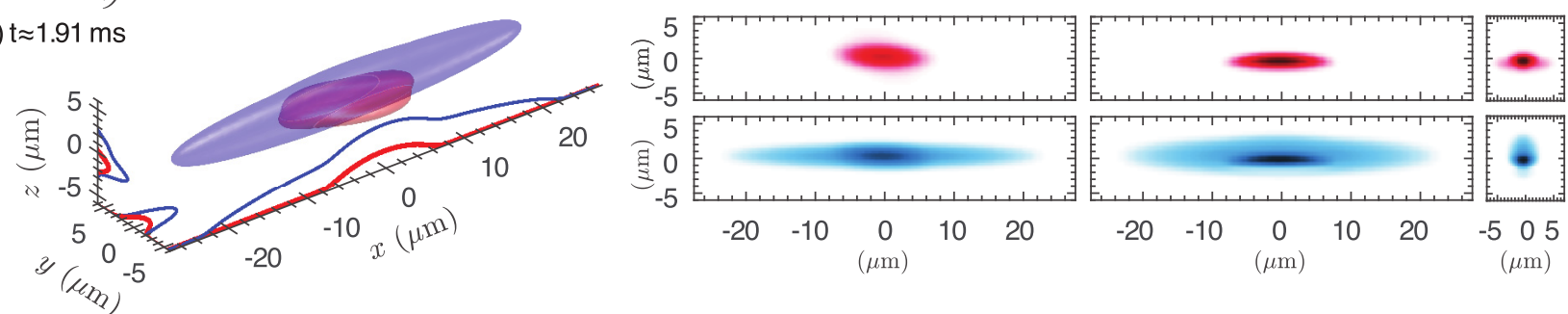

(c) $\mathrm{t} \approx 3.98 \mathrm{~ms}$
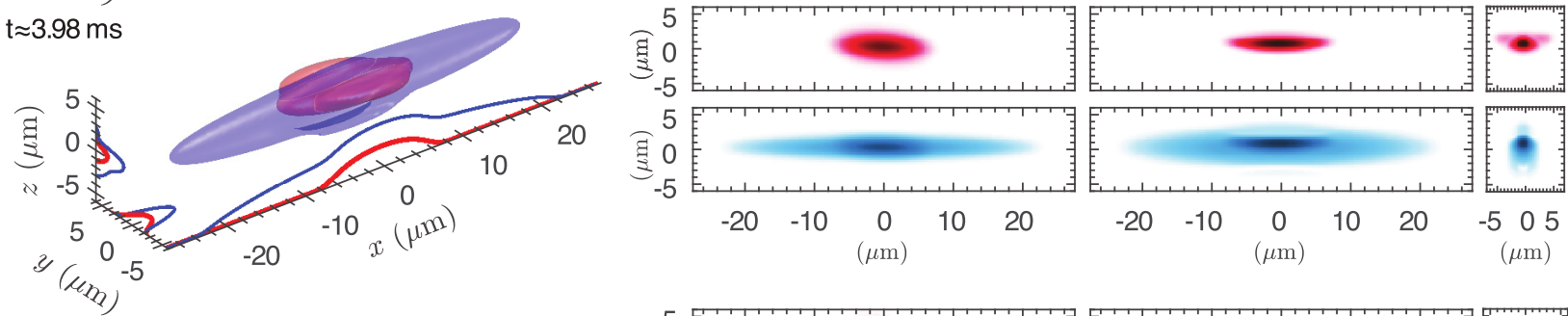

(d) $\mathrm{t} \approx 9.07 \mathrm{~ms}$
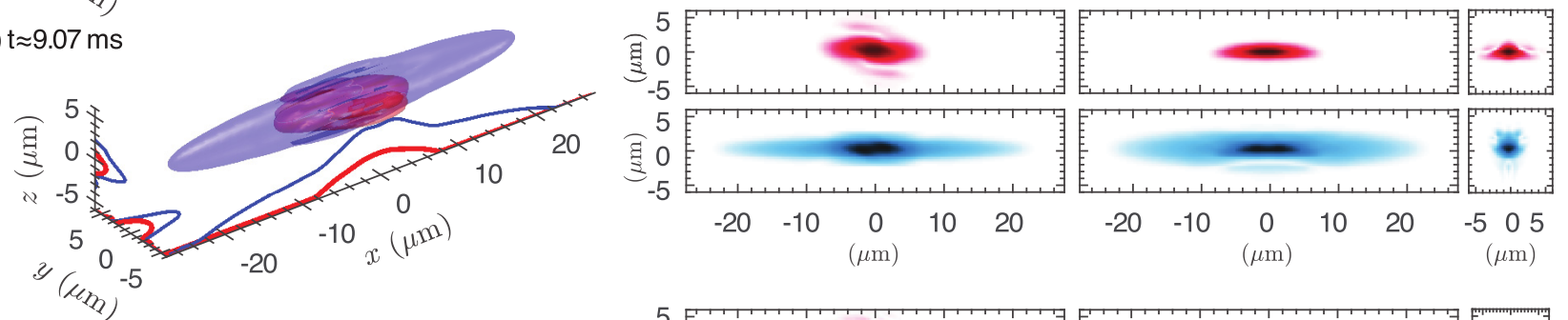

(e) $\mathrm{t} \approx 15.12 \mathrm{~ms}$
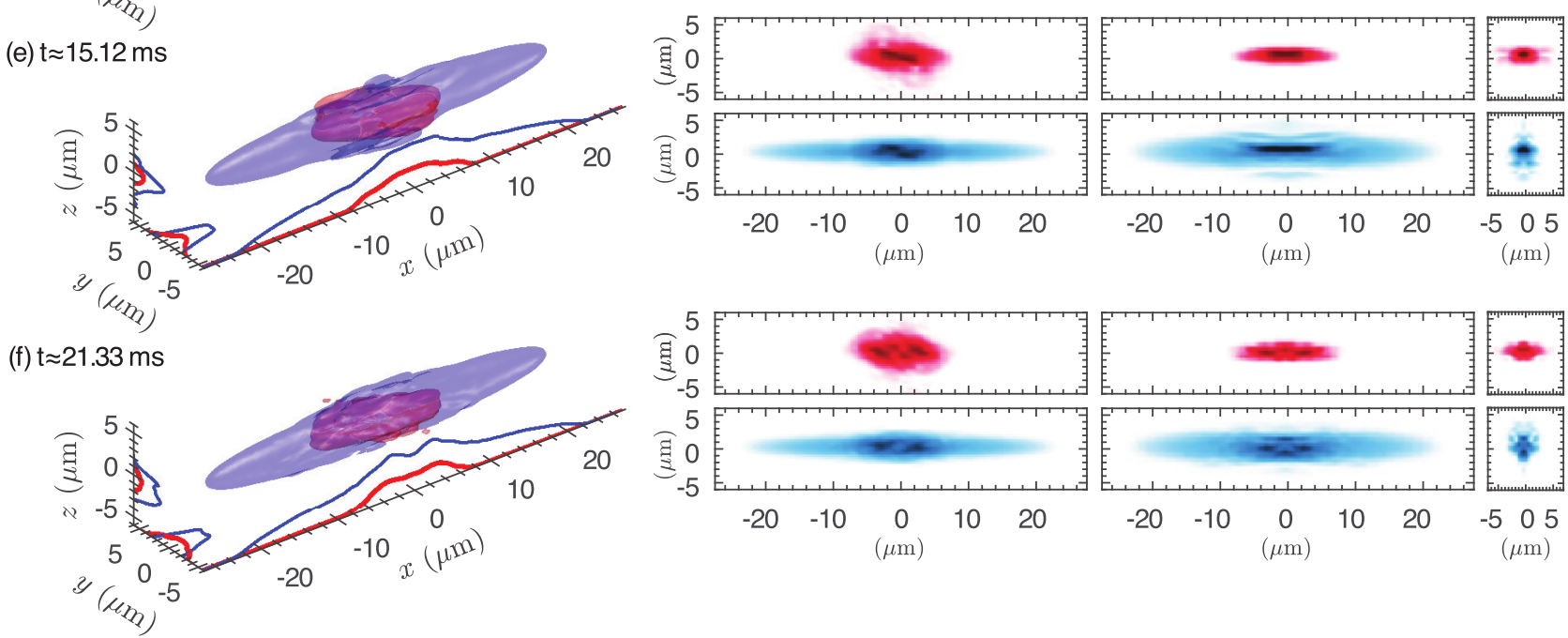

FIG. 7. (a)-(f) Density snapshots of Cs (red) and Yb (blue) at different times during the first $\approx 21 \mathrm{~ms}$ of coupled evolution. Shown are (i) $3 \mathrm{D}$ profiles corresponding to density isosurfaces for the 5\% peak density of $\mathrm{Yb}$ at $t=0$ with corresponding slices shown along each axis and 2D sliced density snapshots across the (ii) $x-y(z=0)$ plane, (iii) $x-z(y=0)$ plane, and (iv) $y-z(x=0)$ plane.

$30 \mathrm{~ms}$, a 10-ms ramp back to $a_{\mathrm{Cs}}(t)=147 a_{0}$, and a final 1-ms hold at this value. At this time in the experimental protocol a time-of-flight measurement begins; in the simulations we simply read out the final atom numbers. We repeat this process for varying values of $a_{\mathrm{Cs}}^{\text {(jump) }}$, obtaining the results reported in the main text [see Fig. 5(b)]. All parameters are set to experimentally measured values rather than fitted to the data, except for the Cs three-body loss coefficient. The latter is less precisely constrained by experimental measurements. In practice we repeated the simulations for a few trial values and found 
that $K_{3, \mathrm{Cs}}=1.8 \times 10^{-27} \mathrm{~cm}^{6} / \mathrm{s}$ gives a reasonable quantitative match to the results of the collapse experiment. As stated in the main text, this is in reasonable agreement with other experimental measurements of $K_{3, \mathrm{Cs}}[28,29,45]$. For reference, Ref. [45] found $8.2 \times 10^{-29} \mathrm{~cm}^{6} / \mathrm{s} \lesssim K_{3, \mathrm{Cs}} \lesssim 1.3 \times 10^{-27}$ $\mathrm{cm}^{6} / \mathrm{s}$ over the range of scattering lengths in our collapse experiments (not including possible calibration uncertainties mentioned therein), Ref. [28] found $1_{-0.9}^{+1} \times 10^{-27} \mathrm{~cm}^{6} / \mathrm{s}$, and Ref. [29] found an upper bound of $\sim 8 \times 10^{-27} \mathrm{~cm}^{6} / \mathrm{s}$. While a direct comparison is difficult because these measurements were performed at different magnetic field strengths and temperature ranges, and these two parameters strongly influence the three-body loss coefficient in the vicinity of an Efimov minimum [56], clearly the order of magnitude is in reasonable agreement. We have not searched for a precise best-fit value of $K_{3, \text { Cs }}$ (e.g., by least-squares fitting) because of the long computational times needed for the simulations.
By default our data points are from simulations in a box of dimension $\left(L_{x}, L_{y}, L_{z}\right)=(96,24,24) \mu \mathrm{m}$ with grid resolution of $3 / 2$ points $/ \mu \mathrm{m}$ in each direction. We checked convergence of the final $N_{\mathrm{Cs}}$ value to $\approx 1 \%$ by repeating selected simulations at higher resolution ( 3 points $/ \mu \mathrm{m}$ ) and in larger boxes in the most-constrained direction $\left(L_{y}=48 \mu \mathrm{m}\right)$. Our data points with the lowest $a_{\mathrm{Cs}}^{\text {(jump) }}$ value in each simulation were obtained from higher-resolution simulations, for which the final $N_{\mathrm{Cs}}$ value agreed to within $\approx 1 \%$ with the larger-box simulations. For values of $a_{\mathrm{Cs}}^{\text {(jump) }} \lesssim-0.7 a_{0}$ (Cs only) or $\lesssim 51 a_{0}$ $(\mathrm{Cs}+\mathrm{Yb})$ the three-body loss coefficient is not sufficient to prevent a mean-field collapse, even in our higher-resolution simulations. When this occurs, the final Cs number is not converged and changes with the grid resolution. Quantitative modeling of this collapse regime would require a description at either finite temperature, beyond mean field, or both, which is beyond the scope of this work.
[1] H. Takeuchi, S. Ishino, and M. Tsubota, Binary Quantum Turbulence Arising from Countersuperflow Instability in TwoComponent Bose-Einstein Condensates, Phys. Rev. Lett. 105, 205301 (2010).

[2] H. Takeuchi, N. Suzuki, K. Kasamatsu, H. Saito, and M. Tsubota, Quantum Kelvin-Helmholtz instability in phaseseparated two-component Bose-Einstein condensates, Phys. Rev. B 81, 094517 (2010).

[3] C. Hamner, J. J. Chang, P. Engels, and M. A. Hoefer, Generation of Dark-Bright Soliton Trains in Superfluid-Superfluid Counterflow, Phys. Rev. Lett. 106, 065302 (2011).

[4] M. Delehaye, S. Laurent, I. Ferrier-Barbut, S. Jin, F. Chevy, and C. Salomon, Critical Velocity and Dissipation of an Ultracold Bose-Fermi Counterflow, Phys. Rev. Lett. 115, 265303 (2015).

[5] A. A. Norrie, R. J. Ballagh, and C. W. Gardiner, Quantum turbulence and correlations in Bose-Einstein condensate collisions, Phys. Rev. A 73, 043617 (2006).

[6] G. Modugno, M. Modugno, F. Riboli, G. Roati, and M. Inguscio, Two Atomic Species Superfluid, Phys. Rev. Lett. 89, 190404 (2002).

[7] E. Fava, T. Bienaimé, C. Mordini, G. Colzi, C. Qu, S. Stringari, G. Lamporesi, and G. Ferrari, Observation of Spin Superfluidity in a Bose Gas Mixture, Phys. Rev. Lett. 120, 170401 (2018).

[8] F. Ferlaino, R. J. Brecha, P. Hannaford, F. Riboli, G. Roati, G. Modugno, and M. Inguscio, Dipolar oscillations in a quantum degenerate Fermi-Bose atomic mixture, J. Opt. B: Quantum Semiclassical Opt. 5, S3 (2003).

[9] I. Ferrier-Barbut, M. Delehaye, S. Laurent, A. T. Grier, M. Pierce, B. S. Rem, F. Chevy, and C. Salomon, A mixture of Bose and Fermi superfluids, Science 345, 1035 (2014).

[10] R. Roy, A. Green, R. Bowler, and S. Gupta, Two-Element Mixture of Bose and Fermi Superfluids, Phys. Rev. Lett. 118, 055301 (2017).

[11] K. L. Lee, N. B. Jørgensen, L. J. Wacker, M. G. Skou, K. T. Skalmstang, J. J. Arlt, and N. P. Proukakis, Time-of-flight expansion of binary Bose-Einstein condensates at finite temperature, New J. Phys. 20, 053004 (2018).
[12] M. Tylutki, A. Recati, F. Dalfovo, and S. Stringari, Dark-bright solitons in a superfluid Bose-Fermi mixture, New J. Phys. 18, 053014 (2016).

[13] X.-C. Yao, H.-Z. Chen, Y.-P. Wu, X.-P. Liu, X.-Q. Wang, X. Jiang, Y. Deng, Y.-A. Chen, and J.-W. Pan, Observation of Coupled Vortex Lattices in a Mass-Imbalance Bose and Fermi Superfluid Mixture, Phys. Rev. Lett. 117, 145301 (2016).

[14] L. Mingarelli and R. Barnett, Exotic Vortex Lattices in Binary Repulsive Superfluids, Phys. Rev. Lett. 122, 045301 (2019).

[15] K. L. Lee, N. B. Jørgensen, I.-K. Liu, L. Wacker, J. J. Arlt, and N. P. Proukakis, Phase separation and dynamics of two-component Bose-Einstein condensates, Phys. Rev. A 94, 013602 (2016).

[16] C. Ospelkaus, S. Ospelkaus, K. Sengstock, and K. Bongs, Interaction-Driven Dynamics of ${ }^{40} \mathrm{~K}-{ }^{87} \mathrm{Rb}$ Fermion-Boson Gas Mixtures in the Large-Particle-Number Limit, Phys. Rev. Lett. 96, 020401 (2006).

[17] G. Modugno, G. Roati, F. Riboli, F. Ferlaino, R. J. Brecha, and M. Inguscio, Collapse of a degenerate Fermi gas, Science 297, 2240 (2002).

[18] B. J. DeSalvo, K. Patel, G. Cai, and C. Chin, Observation of fermion-mediated interactions between bosonic atoms, Nature (London) 568, 61 (2019).

[19] C. R. Cabrera, L. Tanzi, J. Sanz, B. Naylor, P. Thomas, P. Cheiney, and L. Tarruell, Quantum liquid droplets in a mixture of Bose-Einstein condensates, Science 359, 301 (2018).

[20] G. Semeghini, G. Ferioli, L. Masi, C. Mazzinghi, L. Wolswijk, F. Minardi, M. Modugno, G. Modugno, M. Inguscio, and M. Fattori, Self-Bound Quantum Droplets of Atomic Mixtures in Free Space, Phys. Rev. Lett. 120, 235301 (2018).

[21] C. D'Errico, A. Burchianti, M. Prevedelli, L. Salasnich, F. Ancilotto, M. Modugno, F. Minardi, and C. Fort, Observation of quantum droplets in a heteronuclear bosonic mixture, Phys. Rev. Research 1, 033155 (2019).

[22] A. Guttridge, M. D. Frye, B. C. Yang, J. M. Hutson, and S. L. Cornish, Two-photon photoassociation spectroscopy of CsYb: Ground-state interaction potential and interspecies scattering lengths, Phys. Rev. A 98, 022707 (2018). 
[23] C. Chin, V. Vuletić, A. J. Kerman, S. Chu, E. Tiesinga, P. J. Leo, and C. J. Williams, Precision Feshbach spectroscopy of ultracold $\mathrm{Cs}_{2}$, Phys. Rev. A 70, 032701 (2004).

[24] M. Berninger, A. Zenesini, B. Huang, W. Harm, H.-C. Nägerl, F. Ferlaino, R. Grimm, P. S. Julienne, and J. M. Hutson, Feshbach resonances, weakly bound molecular states, and coupled-channel potentials for cesium at high magnetic fields, Phys. Rev. A 87, 032517 (2013).

[25] S. A. Hopkins, K. Butler, A. Guttridge, S. Kemp, R. Freytag, E. A. Hinds, M. R. Tarbutt, and S. L. Cornish, A versatile dualspecies Zeeman slower for caesium and ytterbium, Rev. Sci. Instrum. 87, 043109 (2016).

[26] S. L. Kemp, K. L. Butler, R. Freytag, S. A. Hopkins, E. A. Hinds, M. R. Tarbutt, and S. L. Cornish, Production and characterization of a dual species magneto-optical trap of cesium and ytterbium, Rev. Sci. Instrum. 87, 023105 (2016).

[27] A. Guttridge, S. A. Hopkins, S. L. Kemp, D. Boddy, R. Freytag, M. P. A. Jones, M. R. Tarbutt, E. A. Hinds, and S. L. Cornish, Direct loading of a large $\mathrm{Yb}$ MOT on the ${ }^{1} \mathrm{~S}_{0} \rightarrow{ }^{3} \mathrm{P}_{1}$ transition, J. Phys. B: At., Mol. Opt. Phys. 49, 145006 (2016).

[28] A. Guttridge, S. A. Hopkins, S. L. Kemp, M. D. Frye, J. M. Hutson, and S. L. Cornish, Interspecies thermalization in an ultracold mixture of $\mathrm{Cs}$ and $\mathrm{Yb}$ in an optical trap, Phys. Rev. A 96, 012704 (2017).

[29] K. E. Wilson, A. Guttridge, J. Segal, and S. L. Cornish, Quantum degenerate mixtures of $\mathrm{Cs}$ and Yb, Phys. Rev. A 103, 033306 (2021).

[30] R. Onofrio and C. Presilla, Reaching Fermi Degeneracy in Two-Species Optical Dipole Traps, Phys. Rev. Lett. 89, 100401 (2002).

[31] See Supplemental Material at http://link.aps.org/supplemental/ 10.1103/PhysRevResearch.3.033096 for video of the coupled dynamics.

[32] M. Abad, A. Recati, S. Stringari, and F. Chevy, Counter-flow instability of a quantum mixture of two superfluids, Eur. Phys. J. D 69, 126 (2015).

[33] Y. Castin, I. Ferrier-Barbut, and C. Salomon, La vitesse critique de Landau d'une particule dans un superfluide de fermions, C. R. Phys. 16, 241 (2015).

[34] S. Laurent, P. Parnaudeau, F. Chevy, and I. Danaila, Nonlinear dynamics of coupled superfluids, arXiv:1904.07040.

[35] W. Weimer, K. Morgener, V. P. Singh, J. Siegl, K. Hueck, N. Luick, L. Mathey, and H. Moritz, Critical Velocity in the BECBCS Crossover, Phys. Rev. Lett. 114, 095301 (2015).

[36] V. P. Singh, W. Weimer, K. Morgener, J. Siegl, K. Hueck, N. Luick, H. Moritz, and L. Mathey, Probing superfluidity of BoseEinstein condensates via laser stirring, Phys. Rev. A 93, 023634 (2016).

[37] C. Pethick and H. Smith, Bose-Einstein Condensation in Dilute Gases (Cambridge University Press, Cambridge, 2002).

[38] C. A. Sackett, J. M. Gerton, M. Welling, and R. G. Hulet, Measurements of Collective Collapse in a Bose-Einstein Condensate with Attractive Interactions, Phys. Rev. Lett. 82, 876 (1999).

[39] J. M. Gerton, D. Strekalov, I. Prodan, and R. G. Hulet, Direct observation of growth and collapse of a Bose-Einstein con- densate with attractive interactions, Nature (London) 408, 692 (2000).

[40] J. L. Roberts, N. R. Claussen, S. L. Cornish, E. A. Donley, E. A. Cornell, and C. E. Wieman, Controlled Collapse of a Bose-Einstein Condensate, Phys. Rev. Lett. 86, 4211 (2001).

[41] E. A. Donley, N. R. Claussen, S. L. Cornish, J. L. Roberts, E. A. Cornell, and C. E. Wieman, Dynamics of collapsing and exploding Bose-Einstein condensates, Nature (London) 412, 295 (2001).

[42] S. L. Cornish, S. T. Thompson, and C. E. Wieman, Formation of Bright Matter-Wave Solitons during the Collapse of Attractive Bose-Einstein Condensates, Phys. Rev. Lett. 96, 170401 (2006).

[43] P. A. Ruprecht, M. J. Holland, K. Burnett, and M. Edwards, Time-dependent solution of the nonlinear Schrödinger equation for Bose-condensed trapped neutral atoms, Phys. Rev. A 51, 4704 (1995).

[44] A. Gammal, T. Frederico, and L. Tomio, Critical number of atoms for attractive Bose-Einstein condensates with cylindrically symmetrical traps, Phys. Rev. A 64, 055602 (2001).

[45] T. Kraemer, M. Mark, P. Waldburger, J. G. Danzl, C. Chin, B. Engeser, A. D. Lange, K. Pilch, A. Jaakkola, H.-C. Nägerl, and R. Grimm, Evidence for Efimov quantum states in an ultracold gas of caesium atoms, Nature (London) 440, 315 (2006).

[46] http://dx.doi.org/10.15128/r2bk128991d.

[47] E. A. Burt, R. W. Ghrist, C. J. Myatt, M. J. Holland, E. A. Cornell, and C. E. Wieman, Coherence, Correlations, and Collisions: What One Learns about Bose-Einstein Condensates from Their Decay, Phys. Rev. Lett. 79, 337 (1997).

[48] M. Modugno, L. Pricoupenko, and Y. Castin, Bose-Einstein condensates with a bent vortex in rotating traps, Eur. Phys. J. D 22, 235 (2003).

[49] T. Miyakawa, T. Suzuki, and H. Yabu, Sum-rule approach to collective oscillations of a boson-fermion mixed condensate of alkali-metal atoms, Phys. Rev. A 62, 063613 (2000).

[50] Y. Asano, S. Watabe, and T. Nikuni, Dipole mode of trapped Bose-Fermi mixture gas, J. Low Temp. Phys. 201, 58 (2020).

[51] Y. Asano, S. Watabe, and T. Nikuni, Dipole oscillation of a trapped Bose-Fermi-mixture gas in collisionless and hydrodynamic regimes, Phys. Rev. A 101, 013611 (2020).

[52] P. A. Altin, G. R. Dennis, G. D. McDonald, D. Döring, J. E. Debs, J. D. Close, C. M. Savage, and N. P. Robins, Collapse and three-body loss in a ${ }^{85} \mathrm{Rb}$ Bose-Einstein condensate, Phys. Rev. A 84, 033632 (2011).

[53] G. R. Dennis, J. J. Hope, and M. T. Johnsson, XMDS2: Fast, scalable simulation of coupled stochastic partial differential equations, Comput. Phys. Commun. 184, 201 (2013).

[54] N. G. Parker, S. L. Cornish, C. S. Adams, and A. M. Martin, Bright solitary waves and trapped solutions in Bose-Einstein condensates with attractive interactions, J. Phys. B: At., Mol. Opt. Phys. 40, 3127 (2007).

[55] T. P. Billam, S. A. Wrathmall, and S. A. Gardiner, Variational determination of approximate bright matter-wave soliton solutions in anisotropic traps, Phys. Rev. A 85, 013627 (2012).

[56] J. P. D’Incao, H. Suno, and B. D. Esry, Limits on Universality in Ultracold Three-Boson Recombination, Phys. Rev. Lett. 93, 123201 (2004). 